\title{
The Hilbert-Kunz Function for Binomial Hypersurfaces
}

\author{
Shyamashree Upadhyay \\ Department of Mathematics, Indian Institute of Technology, Guwahati, Assam 781039, India \\ Correspondence should be addressed to Shyamashree Upadhyay; shyamashree.upadhyay@gmail.com
}

Received 6 August 2014; Revised 18 October 2014; Accepted 21 October 2014; Published 27 November 2014

Academic Editor: Prasanna Kumar Sahoo

Copyright (C) 2014 Shyamashree Upadhyay. This is an open access article distributed under the Creative Commons Attribution License, which permits unrestricted use, distribution, and reproduction in any medium, provided the original work is properly cited.

I give an iterative closed form formula for the Hilbert-Kunz function for any binomial hypersurface in general, over any field of arbitrary positive characteristic. I prove that the Hilbert-Kunz multiplicity associated with any binomial hypersurface over any field of arbitrary positive characteristic is rational. As an example, I also prove the well known fact that for 1-dimensional binomial hypersurfaces the Hilbert-Kunz multiplicity is a positive integer and give a precise account of the integer.

\section{Introduction}

Let $(A, \mathfrak{n})$ be a Noetherian local ring of dimension $d$ and of prime characteristic $p>0$. Let $I$ be an $\mathfrak{n}$-primary ideal and $q=p^{n}$ where $n>0$ is an integer. The "Hilbert-Kunz function" of $A$ with respect to $I$ is defined as

$$
H K_{I, A}(q)=l\left(\frac{A}{I^{[q]}}\right)
$$

where $I^{[q]}=n$th Frobenious power of $I$, that is, the ideal generated by $x^{q}, x \in I$.

The associated Hilbert-Kunz multiplicity is defined to be

$$
e_{H K}(I)=\lim _{q \rightarrow \infty} \frac{H K_{I, A}(q)}{q^{d}} \text {. }
$$

Monsky showed in his paper [1] that the limit exists and is a real constant. Further he showed that

$$
H K_{I, A}(q)=e_{H K}(I) q^{d}+O\left(q^{d-1}\right) .
$$

Several authors have investigated $e_{H K}(I)$. They showed that $e_{H K}(I)$ is a rational number for certain special kind of binomial hypersurfaces [2], cubic curves and surfaces [3] and full flag varieties, and elliptic curves [4].

A binomial hypersurface is defined in the beginning of Section 4. In this paper, we are interested in giving an iterative closed form formula for the Hilbert-Kunz function for any binomial hypersurface in general. Our methods work for any positive characteristic. Our work generalizes the work of Conca [2]. In [2], Conca computes the Hilbert-Kunz function of monomial ideals and also of those Binomial hypersurfaces whose terms defining the hypersurface are relatively prime. In [2], Conca also proves that the Hilbert-Kunz multiplicity associated with these special Binomial Hypersurfaces is always rational. In this paper, we prove that the Hilbert-Kunz multiplicity associated with any binomial hypersurface over any field of arbitrary positive characteristic is rational. The rationality result appears in Eto's work (Theorem 2.2, [5]), where the multiplicity is interpreted as a volume of a polytope. Here we give a different proof for the rationality result. Our work also generalizes the work [6] of Watanabe, where he deals only with normal toric varieties.

The organization of this work is more or less clear from the table of contents. But to be precise, until Section 4 begins, the matter of this work holds true for any hypersurface over any field of positive characteristic and need not have to be a binomial hypersurface! Also, the filtration introduced in Section 2.1 is effective for any general ideal $J$ of a polynomial ring $S=k\left[x_{1}, \ldots, x_{m}\right]$ where $k$ is a field of arbitrary prime characteristic $p>0$, not just when the ideal $J$ is generated by a single polynomial. The iterative closed form formula for the Hilbert-Kunz function for binomial hypersurfaces appears in Theorem 26 in Section 4.2.5. After that, in Section 4.4, we discuss the example of the 1-dimensional case. In the example of the 1-dimensional case, I prove the well known 
fact that for any 1-dimensional binomial hypersurface, the associated Hilbert-Kunz multiplicity is a positive integer and I give a precise account of the integer. In Section 4.5 , I give an expression for the Hilbert-Kunz multiplicity for a general binomial hypersurface (see (67)).

\section{Stating the Problem}

For the rest of this paper we will assume that $S=$ $k\left[x_{1}, \ldots, x_{m}\right]$ where $k$ is a field of arbitrary prime characteristic $p>0$ and $J$ is an arbitrary ideal in $S$. Let $R=S / J$. Put $\widehat{\mathfrak{m}}:=\mathfrak{m}+J$ where $\mathfrak{m}=\left(x_{1}, \ldots, x_{m}\right)$. We will assume that $J \subseteq \mathfrak{m}$; otherwise, $\widehat{\mathfrak{m}}=R$. Consider

$$
H K_{\widehat{\mathfrak{m}} R_{\widehat{\mathfrak{m}}}, R_{\widehat{\mathfrak{m}}}}\left(p^{n}\right)=l\left(\frac{R_{\widehat{\mathfrak{m}}}}{\left(\widehat{\mathfrak{m}} R_{\widehat{\mathfrak{m}}}\right)^{\left[p^{n}\right]}}\right) .
$$

Note that the rings $R_{\widehat{\mathfrak{m}}} /\left(\widehat{\mathfrak{m}} R_{\widehat{\mathfrak{m}}}\right)^{\left[p^{n}\right]}$ and $R /\left(\mathfrak{m}^{\left[p^{n}\right]}+J\right)$ are isomorphic, where $\mathfrak{m}^{\left[p^{n}\right]}=\left(x_{1}^{p^{n}}, \ldots, x_{m}^{p^{n}}\right)$. Hence the HilbertKunz function $H K_{\widehat{\mathrm{m}} R_{\widehat{\mathrm{m}}}, R_{\widehat{\mathrm{m}}}}\left(p^{n}\right)$ is given by

$$
H K_{\widehat{\mathfrak{m}} R_{\widehat{\mathfrak{m}}}, R_{\widehat{\mathfrak{m}}}}\left(p^{n}\right)=l\left(\frac{R}{\mathfrak{m}^{\left[p^{n}\right]}+J}\right) .
$$

We are presently interested in the case when $R$ is a hypersurface, that is, when the ideal $J$ is generated by a single polynomial.

2.1. A Filtration for Computing the Length $l\left(R /\left(\mathfrak{m}^{\left[p^{n}\right]}+J\right)\right)$. Let $\mathbb{S}$ denote the set of all $m$-tuples $\left(u_{1}, \ldots, u_{m}\right)$ such that for each $i \in\{1, \ldots, m\}, u_{i} \in\left\{0,1, \ldots, p^{n}-1\right\}$. Let us arrange the elements of the set $\mathbb{\mathfrak { S }}$ in the lexicographic order; that is,

we say that $\left(u_{1}, \ldots, u_{m}\right)<_{\operatorname{lex}}\left(u_{1}^{\prime}, \ldots, u_{m}^{\prime}\right)$ if in the vector difference $\left(u_{1}^{\prime}, \ldots, u_{m}^{\prime}\right)-\left(u_{1}, \ldots, u_{m}\right)$, the leftmost nonzero entry is $>0$.

It is easy to check that the set $\mathbb{\mathfrak { S }}$ equipped with the lexicographic order $<_{\text {lex }}$ is in fact a totally ordered set, and this set contains $p^{m n}$ many elements. Let us denote these $p^{m n}$ many elements of the set $\mathbb{S}$ arranged in the $<_{\operatorname{lex}}$ order by $\mathbb{U}_{0}<_{\text {lex }} \mathbb{U}_{1}<_{\text {lex }} \mathbb{U}_{2}<_{\text {lex }} \cdots<_{\text {lex }} \mathbb{U}_{p^{m n}-1}$.

Let $\mathfrak{M}:=\left\{x_{1}^{j_{1}} \cdots x_{m}^{j_{m}} \mid 0 \leq j_{i}<p^{n} \forall 1 \leq i \leq m\right\}$. There are $p^{m n}$ many elements in the set $\mathfrak{M}$. For any $\mathbb{U}=$ $\left(u_{1}, u_{2}, \ldots, u_{m}\right) \in \mathbb{S}$, let us denote by $\mathbb{X}^{p^{n}-1-\mathbb{U}}$ the element of $\mathfrak{M}$ given by $x_{1}^{p^{n}-1-u_{1}} x_{2}^{p^{n}-1-u_{2}} \cdots x_{m}^{p^{n}-1-u_{m}}$. The set $\mathbb{S}$ is in one-to-one correspondence with the set $\mathfrak{M}$ via the map $\mathbb{U} \mapsto$ $\mathbb{X}^{p^{n}-1-\mathbb{U}}$. Let us denote by $\mathbb{X}^{p^{n}-1-\mathbb{U}_{j}}$ the image of the element $\mathbb{U}_{j}$ under this correspondence.

For any $i \in\{1, \ldots, m\}$ and any $\mathbb{U}_{j}=\left(u_{j, 1}, \ldots, u_{j, m}\right) \in \mathbb{S}$, let us denote by $\mathbb{X}_{i}^{p^{n}-1-\mathbb{U}_{j}}$ the product $x_{1}^{p^{n}-1-u_{j, 1}} \cdots x_{i}^{p^{n}-1-u_{j, i}}$, that is, the product in $\mathbb{X}^{p^{n}-1-\mathbb{U}_{j}}$ up to the $i$ th variable. define

Given any $j \in\left\{0, \ldots, p^{m n}-1\right\}$ and any $i \in\{1, \ldots, m\}$,

$$
\mathscr{B}_{j, i}:= \begin{cases}\mathbb{X}_{i}^{p^{n}-1-\mathbb{U}_{j}} x_{i} & \text { if } u_{j, i}>0 \\ 0 & \text { otherwise. }\end{cases}
$$

Definition 1. The ideals $I_{j}$ : for any $j \in\left\{0, \ldots, p^{m n}-1\right\}$, let $I_{j}:=\left\langle\mathscr{B}_{j, i} \mid 1 \leq i \leq m\right\rangle+L$ where $L=\left\langle x_{1}^{p^{n}}, x_{2}^{p^{n}}, \ldots, x_{m}^{p^{n}}\right\rangle$.

Declare $I_{p^{m n}}$ to be the polynomial ring $S$ itself.

Remark 2. From the definition of the lexicographic order $<_{\text {lex }}$ on $\mathfrak{S}$, it follows easily that for any $j \in\left\{0, \ldots, p^{m n}-2\right\}$, either $\mathbb{X}^{p^{n}-1-\mathbb{U}_{j}}=\mathbb{X}^{p^{n}-1-\mathbb{U}_{j+1}} x_{m}$ or there exists $k \in\{1, \ldots, m-1\}$ such that $\mathbb{X}_{k}^{p^{n}-1-\mathbb{U}_{j}}=\mathbb{X}_{k}^{p^{n}-1-\mathbb{U}_{j+1}} x_{k}, \mathbb{X}^{p^{n}-1-\mathbb{U}_{j}}=\mathbb{X}_{k}^{p^{n}-1-\mathbb{U}_{j}}$, and $\mathbb{X}^{p^{n}-1-\mathbb{U}_{j+1}}=\mathbb{X}_{k}^{p^{n}-1-\mathbb{U}_{j+1}}\left(x_{k+1} \cdots x_{m}\right)^{p^{n}-1}$.

Notation 1. For any $j \in\left\{0, \ldots, p^{m n}-1\right\}$, let $i(j):=\max \{i \mid$ $\left.1 \leq i \leq m, \mathscr{B}_{j, i} \neq 0\right\}$.

Lemma 3. (i) For any $j \in\left\{0, \ldots, p^{m n}-1\right\}, \mathbb{X}^{p^{n}-1-\mathbb{U}_{j}} \in I_{j+1}$;

(ii) $\mathscr{B}_{j, k}=\mathscr{B}_{j+1, k}$ for all $j \in\left\{0, \ldots, p^{m n}-2\right\}$ and for all $k<i(j+1)$.

Proof. (i) It follows from Remark 2 that $\mathbb{X}^{p^{n}-1-\mathbb{U}_{j}}=$ $\mathscr{B}_{j+1, i(j+1)}$. Hence $\mathbb{X}^{p^{n}-1-\mathbb{U}_{j}} \in I_{j+1}$;

(ii) the proof of this follows from Remark 2.

Lemma 4. For any $j \in\left\{0, \ldots, p^{m n}-1\right\}, I_{j} \subseteq I_{j+1}$.

Proof. As $I_{j}:=\left\langle\mathscr{B}_{j, i} \mid 1 \leq i \leq m\right\rangle+L$ (where $L=$ $\left.\left\langle x_{1}^{p^{n}}, x_{2}^{p^{n}}, \ldots, x_{m}^{p^{n}}\right\rangle\right)$, it is enough to show that $\mathscr{B}_{j, i} \in I_{j+1}$ for all $j \in\left\{0, \ldots, p^{m n}-1\right\}$ and for all $i \in\{1, \ldots, m\}$.

Case 1 (when $\mathbb{X}^{p^{n}-1-\mathbb{U}_{j}}=\mathbb{X}^{p^{n}-1-\mathbb{U}_{j+1}} x_{m}$ ). In this case, clearly $\mathscr{B}_{j, i}=\mathscr{B}_{j+1, i}$ for all $1 \leq i \leq m-1$. And $\mathscr{B}_{j, m}$ is either equal to 0 or equal to $\mathscr{B}_{j+1, m} x_{m}$. Hence in either case, $\mathscr{B}_{j, m} \in I_{j+1}$. Therefore, $I_{j} \subseteq I_{j+1}$.

Case 2 (when $\mathbb{X}^{p^{n}-1-\mathbb{U}_{j}} \neq \mathbb{X}^{p^{n}-1-\mathbb{U}_{j+1}} x_{m}$ ). In this case, there exists $k \in\{1, \ldots, m-1\}$ such that $\mathbb{X}_{k}^{p^{n}-1-\mathbb{U}_{j}}=$ $\mathbb{X}_{k}^{p^{n}-1-\mathbb{U}_{j+1}} x_{k}, \mathbb{X}^{p^{n}-1-\mathbb{U}_{j}}=\mathbb{X}_{k}^{p^{n}-1-\mathbb{U}_{j}}$, and $\mathbb{X}^{p^{n}-1-\mathbb{U}_{j+1}}=$ $\mathbb{X}_{k}^{p^{n}-1-\mathbb{U}_{j+1}}\left(x_{k+1} \cdots x_{m}\right)^{p^{n}-1}$.

Clearly then, $\mathscr{B}_{j, i}=\mathscr{B}_{j+1, i}$ for all $i<k$. Also, $\mathscr{B}_{j, k}$ is either equal to 0 or equal to $\mathscr{B}_{j+1, k} x_{k}$. Hence in either case, $\mathscr{B}_{j, k} \in I_{j+1}$. For $i>k, \mathscr{B}_{j, i}=\mathscr{B}_{j+1, k} x_{i}$. Hence $\mathscr{B}_{j, i} \in I_{j+1}$ for all $i>k$. Therefore, $I_{j} \subseteq I_{j+1}$.

Notation 2. Let $M$ be any $S$-module. Let $\lambda(M)$ denote the length of $M$ with respect to $S$.

Lemma 5. Let $j \in\left\{0, \ldots, p^{m n}-1\right\}$. Put $M_{j+1}:=I_{j+1} / I_{j}$. Then $\lambda\left(M_{j+1}\right)=1$.

Proof. Fix $j$. We will show that for each $i \in\{1, \ldots, m\}, x_{i} I_{j+1} \subseteq$ $I_{j}$. We claim that for any $i, k \in\{1, \ldots, m\}, x_{i} \mathscr{B}_{j+1, k} \in I_{j}$.

Case 1 (when $k>i(j+1)$ ). In this case, we have $\mathscr{B}_{j+1, k}=0$. Hence $x_{i} \mathscr{B}_{j+1, k} \in I_{j}$ for any $k>i(j+1)$ and for any $i \in$ $\{1, \ldots, m\}$.

Case 2 (when $k \leq i(j+1)$ ). It follows from Lemma 3 that $\mathscr{B}_{j, k}=\mathscr{B}_{j+1, k}$ for all $k<i(j+1)$. Therefore $\mathscr{B}_{j+1, k} \in I_{j}$ for all 
$k<i(j+1)$. This implies that $x_{i} \mathscr{B}_{j+1, k} \in I_{j}$ for all $k<i(j+1)$ and for all $i \in\{1, \ldots, m\}$.

It remains to show that $x_{i} \mathscr{B}_{j+1, i(j+1)} \in I_{j}$ for all $i \in$ $\{1, \ldots, m\}$. It follows from the proof of Lemma 3(i) that $\mathbb{X}^{p^{n}-1-\mathbb{U}_{j}}=\mathscr{B}_{j+1, i(j+1)}$. Hence for any $i \in\{1, \ldots, m\}$, $x_{i} \mathscr{B}_{j+1, i(j+1)}=x_{i} \mathbb{X}^{p^{n}-1-\bigcup_{j}}$ is either a multiple of $\mathscr{B}_{j, i}$ or a multiple of $x_{i}^{p^{n}}$. Therefore $x_{i} \mathscr{B}_{j+1, i(j+1)} \in I_{j}$ for all $i \epsilon$ $\{1, \ldots, m\}$.

Lemma 6. For all $j \in\left\{0, \ldots, p^{m n}-1\right\}, I_{j+1}=\left\langle\mathbb{X}^{p^{n}-1-\bigcup_{j}}\right\rangle+I_{j}$. Proof. It follows from Lemma 3 that $\mathbb{X}^{p^{n}-1-\mathbb{U}_{j}} \in I_{j+1}$ for all $j \in\left\{0, \ldots, p^{m n}-1\right\}$. It also follows from the same lemma that $\mathscr{B}_{j, k}=\mathscr{B}_{j+1, k}$ for all $j \in\left\{0, \ldots, p^{m n}-2\right\}$ and for all $k<i(j+1)$. Therefore the generators $\mathscr{B}_{j+1, k}$ (where $k \neq i(j+1)$ ) of the ideal $I_{j+1}$ belong to $I_{j}$. Due to degree reasons, $\mathbb{X}^{p^{n}-1-U_{j}}=$ $\mathscr{B}_{j+1, i(j+1)}$ does not belong to $I_{j}$. Therefore $\mathbb{X}^{p^{n}-1-\mathbb{U}_{j}}$ is the only generator of the ideal $I_{j+1}$ which does not belong to $I_{j}$. Hence $I_{j+1}=\left\langle\mathbb{X}^{p^{n}-1-\mathbb{U}_{j}}\right\rangle+I_{j}$.

It follows from Lemmas 4, 5, and 6 above that the following chain

$$
I_{0} \subsetneq I_{1} \subsetneq \cdots \subsetneq I_{p^{n n}-1} \subsetneq I_{p^{m n}}
$$

is a filtration of ideals such that $\lambda\left(I_{j+1} / I_{j}\right)=1$.

Therefore reducing modulo the ideal $J$, it follows that the following chain

$$
\begin{aligned}
(0) & =\frac{I_{0}+J}{I_{0}+J} \subseteq \frac{I_{1}+J}{I_{0}+J} \subseteq \frac{I_{2}+J}{I_{0}+J} \subseteq \cdots \subseteq \frac{I_{p^{m n}}+J}{I_{0}+J} \\
& =\frac{R}{\mathfrak{m}^{\left[p^{n}\right]}+J}
\end{aligned}
$$

is a filtration of ideals, which is having the property that each successive quotient either is zero or is generated by a single element. The total number of successive quotients of the above mentioned filtration which are generated by a single element equals the length $l\left(R /\left(\mathfrak{m}^{\left[p^{n}\right]}+J\right)\right)$. The following corollary to Lemma 6 above gives a precise account of which successive quotient of the above mentioned filtration is generated by a single element and which successive quotient is zero.

Corollary 7. For any $j \in\left\{0, \ldots, p^{m n}-1\right\}, \lambda\left(\left(I_{j+1}+J\right) /\left(I_{j}+\right.\right.$ $J)) \leq 1$, and $\lambda\left(\left(I_{j+1}+J\right) /\left(I_{j}+J\right)\right)=0$ if and only if $\mathbb{X}^{p^{n}-1-U_{j}} \in$ $I_{j}+J$.

Proof. Fix any $j \in\left\{0, \ldots, p^{m n}-1\right\}$, it follows from Lemma 5 that $\lambda\left(I_{j+1} / I_{j}\right)=1$. Therefore, reducing modulo the ideal $J$, we have $\lambda\left(\left(I_{j+1}+J\right) /\left(I_{j}+J\right)\right) \leq 1$. Let $M_{j+1}$ be as in Lemma 5. It follows from Lemma 6 that $\mathbb{X}^{p^{n}-1-\mathbb{U}_{j}}$ generates $M_{j+1}$. Therefore reducing modulo the ideal $J$, it follows that the quotient $\left(I_{j+1}+J\right) /\left(I_{j}+J\right)$ is zero if and only if $\mathbb{X}^{p^{n}-1-\mathbb{U}_{j}} \in I_{j}+J$ and is generated by the single element $\mathbb{X}^{p^{n}-1-\mathbb{U}_{j}}$ otherwise.
Hence for knowing that which successive quotient of the above mentioned filtration is generated by single element and which one is zero, the only condition that needs to be verified is the following:

$$
\mathbb{X}^{p^{n}-1-\mathbb{U}_{j}} \in I_{j}+J \text { or not for every } j \in\left\{0, \ldots, p^{m n}-1\right\} \text { ? }
$$

This is the key checking condition for computing the length $l\left(R /\left(\mathfrak{m}^{\left[p^{n}\right]}+J\right)\right)$.

\section{A Procedure for Doing the Key Check}

We are interested in the case when the ideal $J$ is generated by a single polynomial, say $J=(f)$. In this section, we will first define a term order $\triangleright$ on the set of all monomials in the variables $x_{1}, \ldots, x_{m}$, and then with respect to $\triangleright$, we will arrange the terms of the polynomial $f$, and with the help of all this notation, we will describe a way of doing the key checking.

3.1. The Term Order $\triangleright$. To order the terms of the polynomial $f$, let us put an order (denote it by $\triangleright$ ) on the set of all monomials in the variables $x_{1}, \ldots, x_{m}$ as follows.

(i) Set $x_{1} \triangleright \cdots \triangleright x_{m}$.

(ii) On the set of all monomials in the variables $x_{1}, \ldots, x_{m}, \triangleright$ is the degree lexicographic order with respect to the order $\triangleright$ defined on the variables $x_{1}, \ldots, x_{m}$.

Say, the polynomial $f$ has $F$ many terms. Let us denote the most initial (with respect to $\triangleright$ ) term of $f$ by $[F]$, the next most initial term of $f$ by $[F-1], \ldots$, and so on till the least initial term [1]. Hence we have

$$
J=(f)=([F]+[F-1]+\cdots+[1]) .
$$

For example, when $m=5$ and $f=x_{1}^{3}+x_{2}^{3}+x_{1} x_{2} x_{3}+x_{4} x_{5}$, then $F=4,[4]=x_{1}^{3},[3]=x_{1} x_{2} x_{3},[2]=x_{2}^{3}$, and [1] $=x_{4} x_{5}$.

Remark 8. Note here that the terms $[F],[F-1], \ldots,[1]$, of $f$ are assumed to be containing scalar coefficients.

3.2. The Main Theorem. Given any term $[\tau]$ of the polynomial $f$, define $[\tau]^{-1}:=1 /[\tau]$. Recall the set $\left\{\mathfrak{M}:=\mathbb{X}^{p^{n}-1-\mathbb{U}_{j}} \mid j \in\right.$ $\left.\left\{0, \ldots, p^{m n}-1\right\}\right\}$. Let $A$ be an arbitrary element of the set $\mathfrak{M}$. Say the monomial $A$ chosen above from the set $\mathfrak{M}$ equals $\mathbb{X}^{p^{n}-1-\mathbb{U}_{j}}$ for some $j \in\left\{0, \ldots, p^{m n}-1\right\}$. The key checking condition for the monomial $A$ says that " $A \in I_{j}+J$ or not." Let us denote by $A_{c}$ the ideal $I_{j}$. We call $A_{c}$ the ideal of convergence corresponding to $A$. Given any $A \in \mathfrak{M}$, we need to check whether or not $A \in A_{c}+J$. Theorem 10 below provides a way for doing this checking.

Definition 9. A monomial in the variables $x_{1}, \ldots, x_{m}$ of the type

$$
A\left[\tau_{1}\right]^{-1} \cdots\left[\tau_{r}\right]^{-1}\left[\alpha_{1}\right] \cdots\left[\alpha_{r}\right],
$$


where $\left[\tau_{i}\right]$ and $\left[\alpha_{i}\right]$ are terms of the polynomial $f$ and $r$ is any nonnegative integer, is called a combination monomial in $A$ and $f$ if $A\left[\tau_{1}\right]^{-1} \cdots\left[\tau_{r}\right]^{-1}\left[\alpha_{1}\right] \cdots\left[\alpha_{r}\right]$ contains no negative powers of any of the underlying variables $x_{1}, \ldots, x_{m}$. In particular, the monomial $A$ itself is a combination monomial in $A$ and $f$.

Theorem 10. Let $f=[F]+[F-1]+\cdots+[1] . A \in A_{c}+J$ if and only if one of the following mutually exclusive conditions holds.

(i) The term [1] of $f$ divides the monomial A.

(ii) The term [1] of $f$ does not divide the monomial A, but there exists a polynomial $h$ whose terms are of the type $c B[\tau]^{-1}$ where $c$ is a nonzero scalar, $B$ is a combination monomial in $A$ and $f$ such that $B \notin A_{c},[\tau]$ is a term of $f$ which divides $B$, such that the product $f \cdot h$ equals $a A+$ finitely many terms all belonging to $A_{c}$ where $a$ is a nonzero scalar.

Proof. If condition (i) of the theorem holds, the proof follows easily from the construction of the ideal $A_{c}$. If condition (ii) of the theorem holds, then it is easy to see that $A \in A_{c}+J$.

Conversely, suppose $A \in A_{c}+J$. Note that due to degree reasons, the monomial $A$ can never belong to the ideal $A_{c}$ (the proof of this follows easily from the construction of the ideal $\left.A_{c}\right)$. Therefore the fact that $A \in A_{c}+J$ implies that there exists a polynomial (say $g$ ) such that the product $f \cdot g$ equals $a A+$ finitely many terms all belonging to $A_{c}$ where $a$ is a nonzero scalar in the ground field $k$.

Since the product $f \cdot g$ contains a term of the type $a A$ (for some nonzero scalar $a$ ), therefore the polynomial $g$ contains finitely many terms of the form $c A[\tau]^{-1}$ where $c$ is a nonzero scalar and $[\tau]$ is a term of $f$ such that $[\tau]$ divides $A$. Say $c_{1} A\left[\tau_{1}\right]^{-1}+\cdots+c_{l} A\left[\tau_{l}\right]^{-1}$ is the part of the polynomial $g$ which has all its terms of the form $c A[\tau]^{-1}$ where $c$ is some nonzero scalar and $[\tau]$ is some term of $f$ which divides $A$. If at least one of the $\left[\tau_{i}\right]^{\prime}$ s appearing in the expression $c_{1} A\left[\tau_{1}\right]^{-1}+\cdots+$ $c_{l} A\left[\tau_{l}\right]^{-1}$ is equal to [1], then we can conclude that the term [1] divides $A$ and we are done.

If not, then look at the portion (say, $g_{1}$ ) of the polynomial $g$ which consists of sum of all terms of the type $c B[\tau]^{-1}$ where $c$ is a nonzero scalar, $B$ is a combination monomial in $A$ and $f$ such that $B \notin A_{c}$, and $[\tau]$ is a term of $f$ which divides $B$. Clearly then $g_{1}$ contains the sum $c_{1} A\left[\tau_{1}\right]^{-1}+\cdots+c_{l} A\left[\tau_{l}\right]^{-1}$. If $f \cdot g_{1}$ equals $a A+$ finitely many terms all belonging to $A_{c}$ for some nonzero scalar $a$, then condition (ii) of the theorem holds and with $h=g_{1}$ and we are done.

So assume that the product $f \cdot g_{1}$ contains at least one term (say, $D_{1}$ ) which is a combination monomial in $A$ and $f$ not belonging to $A_{c}$. Since the product $f \cdot g$ equals $a A+$ finitely many terms all belonging to $A_{c}$ for some nonzero scalar $a$, therefore there exists a nonzero term $E$ in $g$ such that $E$ is not a term of the polynomial $g_{1}$ and $E[\tau]=b D_{1}$ for some nonzero scalar $b$ and some term $[\tau]$ of $f$. Hence $E=$ $b D_{1}[\tau]^{-1}$ for some nonzero scalar $b$ and some term $[\tau]$ of $f$ which divides $D_{1}$. But since $D_{1}$ is a combination monomial in $A$ and $f$ not belonging to $A_{c}$, it follows from the equation
$E=b D_{1}[\tau]^{-1}$ that $E$ is a term of the polynomial $g_{1}$, hence a contradiction.

\section{The Case of Binomial Hypersurfaces}

In this section, we will study the case where the polynomial $f$ contains only 2 terms; that is, $f=[2]+[1]$. The affine variety defined by the ideal $J$ where $J=(f)=([2]+[1])$ is called a binomial hypersurface.

4.1. The Main Theorem for Binomial Hypersurfaces. In this subsection, we will prove a theorem which will reduce the "key checking condition" mentioned above to checking of a combinatorial condition. The theorem which does this job is the following.

Theorem 11. $A \in A_{c}+J$ if and only if either [1] divides $A$ or there exists a positive integer $M$ for which $A[2]^{-M}[1]^{M-1}$ contains no negative powers of any of the underlying variables and $A[2]^{-M}[1]^{M}$ belongs to $A_{c}$. (Note here that by the notation $[2]^{-M}$, we mean $[2]^{-1}$ multiplied $M$ times and similarly for the notation $[1]^{M}$.)

Proof. If [1] divides $A$, then it follows from Theorem 10 above that $A \in A_{c}+J$.

Suppose there exists a positive integer $M$ for which $A[2]^{-M}[1]^{M-1}$ has no negative powers and $A[2]^{-M}[1]^{M}$ belongs to $A_{c}$. Let $M_{0}$ denote the least positive integer $M$ for which this happens. Then the polynomial $g$ given by

$$
a_{1} A[2]^{-1}+a_{2} A[2]^{-2}[1]^{1}+\cdots+a_{M_{0}} A[2]^{-M_{0}}[1]^{M_{0}-1},
$$

where $a_{1}$ is a nonzero scalar in the ground field $k$ and $a_{i}+$ $a_{i+1}=0(\bmod p)$ for each $i \in\left\{1, \ldots, M_{0}-1\right\}$, is having the property that the product $f \cdot g$ equals $a A+$ finitely many terms all belonging to $A_{c}$ where $a$ is a nonzero scalar. Hence $A \in A_{c}+J$. We now need to prove the other way round.

Suppose $A \in A_{c}+J$ and [1] does not divide $A$. Then by Theorem 10, there exists a polynomial $h$ whose terms are of the type $c B[\tau]^{-1}$ where $c$ is a nonzero scalar, $B$ is a combination monomial in $A$ and $f$ not belonging to $A_{c}$, and $[\tau]$ is a term of $f$ which divides $B$, such that the product $f \cdot h$ equals $a A+$ finitely many terms all belonging to $A_{c}$ where $a$ is a nonzero scalar.

It is easy to see that for the case of a binomial hypersurface, any combination monomial in $A$ and $f$ is either equal to $A$ itself or it is of the type $A[2]^{-N}[1]^{N}$ for some positive integer $N$ or it is of the type $A[1]^{-N}[2]^{N}$ for some positive integer $N$. It is also elementary to observe that any combination monomial in $A$ and $f$ of the type $A[1]^{-N}[2]^{N}$ always belongs to $A_{c}$. So any combination monomial not belonging to $A_{c}$ is either equal to $A$ itself or of the type $A[2]^{-N}[1]^{N}$ for some positive integer $N$. It hence follows that the terms of the polynomial $h$ are either of the type $A[2]^{-N}[1]^{N-1}$ for some positive integer $N$ or of the type $A[1]^{-1}$. But we have assumed that the term [1] does not divide $A$, so $A[1]^{-1}$ cannot be a term of the polynomial $h$. Hence the 
terms of the polynomial $h$ are only of the type $A[2]^{-N}[1]^{N-1}$ for some positive integer $N$.

Let $N_{0}$ denote the largest positive integer $N$ for which $A[2]^{-N}[1]^{N-1}$ is a term of the polynomial $h$. Clearly then $A[2]^{-N_{0}}[1]^{N_{0}-1}$ has no negative powers of any of the underlying variables $x_{1}, \ldots, x_{m}$. Moreover, since the product $f \cdot h$ equals $a A+$ finitely many terms all belonging to $A_{c}$ (where $a$ is a nonzero scalar), it follows that $A[2]^{-N_{0}}[1]^{N_{0}}$ belongs to $A_{c}$.

4.2. A Formula for the Hilbert-Kunz Function for Binomial Hypersurfaces. In this subsection, we will give a closed form iterative formula for computing the Hilbert-Kunz function for any binomial hypersurface in general, over any field of positive characteristic. The notation and terminology remain the same as in the previous part of this paper.

4.2.1. Notation. Recall from Section 3.1 the variables $x_{1}, \ldots$, $x_{m}$ and the term order $\triangleright$ on the set of all monomials in these variables. Let $f=[2]+[1]$ be the polynomial defining the binomial hypersurface where [2] and [1] are defined as in Section 3.1.

For any $i \in\{1, \ldots, m\}$, let

$$
\begin{aligned}
\Delta_{i}:= & \text { the power of } x_{i} \text { in the term [1] } \\
& - \text { the power of } x_{i} \text { in the term [2] }
\end{aligned}
$$

Note that some of the $\Delta_{i}$ 's can be negative, some can be positive, and some can be 0 . Without loss of generality, we can assume that $\Delta_{1} \leq \cdots \leq \Delta_{m}$.

Let $r$ be the integer such that $0 \leq r \leq m$ and, the ordered set $\left\{x_{1} \triangleright \cdots \triangleright x_{r}\right\}$ equals the set $\left\{x_{i} \mid 1 \leq i \leq m, \Delta_{i}<0\right\}$. For $i \in\{1, \ldots, r\}$, let $N_{i}:=x_{r+1-i}$ and $\Delta_{N_{i}}:=\Delta_{r+1-i}$. Similarly, let $s$ denote the number of $x_{i}$ 's for which $\Delta_{i}=0$ (note that $s$ can also be 0). Let $x_{r+1} \triangleright \cdots \triangleright x_{r+s}$ be the elements of the set $\left\{x_{i} \mid 1 \leq i \leq m, \Delta_{i}=0\right\}$. For $i=\{1, \ldots, s\}$, let $Z_{i}:=$ $x_{r+s+1-i}$ and $\Delta_{Z_{i}}:=\Delta_{r+s+1-i}$. Finally, let $t:=m-(r+s)$ and let $x_{r+s+1} \triangleright \cdots \triangleright x_{m}$ be the elements of the set $\left\{x_{i} \mid 1 \leq i \leq\right.$ $\left.m, \Delta_{i}>0\right\}$. Let us denote by $P_{1} \triangleright \cdots \triangleright P_{t}$ the ordered set $x_{r+s+1} \triangleright \cdots \triangleright x_{m}$ and for $i=\{1, \ldots, t\}$, let $\Delta_{P_{i}}:=\Delta_{r+s+i}$. In other words, we can say that the ordered set $x_{1} \triangleright \cdots \triangleright x_{m}$ is the same as the ordered set $N_{r} \triangleright \cdots \triangleright N_{1} \triangleright Z_{s} \triangleright \cdots \triangleright Z_{1} \triangleright$ $P_{1} \triangleright \cdots \triangleright P_{t}$. We call $N_{i}$ 's the negative difference variables, $Z_{i}$ 's the zero difference variables, and $P_{i}$ 's the positive difference variables. For any $i \in\{1, \ldots, r\}$, let $N_{i, \min }$ and $N_{i, \max }$ denote the minimum and the maximum powers, respectively, of the variable $N_{i}$ that appears in the expression of the polynomial $f$. We can similarly define $P_{q, \min }, P_{q, \max }, Z_{j, \min }$, and $Z_{j, \max }$ for all $q \in\{1, \ldots, t\}$ and for all $j \in\{1, \ldots, s\}$. Observe that since each $Z_{j}$ is a zero difference variable, we have $Z_{j, \text { min }}=Z_{j, \text { max }}$ for all $j \in\{1, \ldots, s\}$.

For any $i \in\{1, \ldots, r\}$, let $a_{i}:=-\left(\Delta_{N_{i}}\right)$ and $b_{i}:=N_{i, \min }$. For any $i \in\{1, \ldots, r\}$ and any positive integer $n$, let $M_{a_{i}, n}$ and $r_{a_{i}, n}$ be defined by the equation $p^{n}-1=a_{i} M_{a_{i}, n}+r_{a_{i}, n}$.

\subsubsection{The Number $M_{\max , A}$}

Definition 12 (of $\left.M_{\max , A}\right)$. Let $A$ be an arbitrary element of the set $\mathfrak{M}$ (this set has been defined earlier) such that [7] divides $A$. It follows from Theorem 11 that for those monomials $A$ which are divisible by [7], we need to keep an account of the largest positive integer $M$ for which the monomial $A[2]^{-M}[1]^{M-1}$ has no negative power for any of the variables $x_{1}, \ldots, x_{m}$. Let $M_{\max , A}$ denote this largest positive integer.

4.2.3. An Explicit Account of $M_{\max , A}$. Corollary 15 below gives an explicit account of the number $M_{\max , A}$, for any monomial $A$ which is divisible by [7]. The number $M_{\max , A}$ is important to us because of Theorem 11 which provides us with a way to count the required length $l\left(R /\left(\mathfrak{m}^{\left[p^{n}\right]}+J\right)\right)$.

Remark 13. Let $A$ be any monomial in $\mathfrak{M}$ which [2] divides; then it is of the form

$$
N_{r}^{p^{n}-k_{N_{r}}} \cdots N_{1}^{p^{n}-k_{N_{1}}} Z_{s}^{p^{n}-k_{Z_{s}}} \cdots Z_{1}^{p^{n}-k_{Z_{1}}} P_{1}^{j_{1}} \cdots P_{t}^{j_{t}},
$$

where $p^{n}-1 \geq j_{q} \geq P_{q, \text { min }}$ for any $q \in\{1, \ldots, t\}, p^{n}-1 \geq p^{n}-$ $k_{N_{i}} \geq N_{i, \text { max }}$ for every $1 \leq i \leq r$, and $p^{n}-1 \geq p^{n}-k_{Z_{i}} \geq Z_{i, \max }$ for every $1 \leq i \leq s$.

Hence for any positive integer $M, A[2]^{-M}[1]^{M-1}$ is of the form

$$
\prod_{i=1}^{r} N_{i}^{p^{n}-k_{N_{i}}-a_{i} M-b_{i}} \prod_{i=1}^{s} Z_{i}^{p^{n}-k_{Z_{i}}-Z_{i, \min }} \prod_{q=1}^{t} P_{q}^{j_{q}+M \Delta_{P_{q}}-P_{q, \max }},
$$

where $p^{n}-1 \geq j_{q} \geq P_{q, \min }$ for any $q \in\{1, \ldots, t\}, p^{n}-1 \geq p^{n}-$ $k_{N_{i}} \geq N_{i, \max }$ for every $1 \leq i \leq r$, and $p^{n}-1 \geq p^{n}-k_{Z_{i}} \geq Z_{i, \max }$ for every $1 \leq i \leq s$.

It is now easy to see that for any $M \geq 1$, the power of any zero difference variable or any positive difference variable in $A[2]^{-M}[1]^{M-1}$ is $\geq 0$. But the power of any negative difference variable in $A[2]^{-M}[1]^{M-1}$ may be negative for some values of $M$.

The following lemma and its corollary give an explicit account of $M_{\text {max }, A}$ for any monomial $A$ in $\mathfrak{M}$ which [2] divides. But for stating the lemma, we need some notation. Let $i \in\{1, \ldots, r\}$ and let $n$ be any positive integer. If $b_{i} \geq a_{i}$, then let $y_{a_{i}}$ denote the least positive integer $\geq\left(b_{i}-r_{a_{i}, n}\right)$ which is divisible by $a_{i}$. Let $q_{a_{i}}$ be defined by the equation $y_{a_{i}}=a_{i} q_{a_{i}}$. Let

$$
p_{a_{i}}:= \begin{cases}0 & \text { if } b_{i}<a_{i} \text { and } r_{a_{i}, n} \geq b_{i}, \\ 1 & \text { if } b_{i}<a_{i} \text { and } r_{a_{i}, n}<b_{i}, \\ q_{a_{i}} & \text { if } b_{i} \geq a_{i}\left[\text { clearly here } r_{a_{i}, n}<b_{i}\right]\end{cases}
$$

And let

$$
E_{a_{i}, b_{i}}:= \begin{cases}r_{a_{i}, n}-b_{i} & \text { if } p_{a_{i}}=0, \\ \left(r_{a_{i}, n}-b_{i}\right)+a_{i} & \text { if } p_{a_{i}}=1, \\ y_{a_{i}}-\left(b_{i}-r_{a_{i}, n}\right) & \text { if } p_{a_{i}}=q_{a_{i}} .\end{cases}
$$


For any real number $\beta$, let $\langle\beta\rangle$ denote the smallest positive integer $\geq \beta$. For any $1 \leq i \leq r$, let

$$
\tilde{k}_{N_{i} \text {, last }}:=\left\langle\frac{p^{n}-N_{i, \max }-1-E_{a_{i}, b_{i}}}{a_{i}}\right\rangle .
$$

Lemma 14. For any $1 \leq i \leq r$, if $k_{N_{i}}$ is such that $1 \leq k_{N_{i}} \leq$ $p^{n}-N_{i, \max }$, then the maximum value of the positive integer $M$ for which $p^{n}-k_{N_{i}}-a_{i} M-b_{i}$ is $\geq 0$ equals

$$
\begin{array}{ccc}
M_{a_{i}, n}-p_{a_{i}} & \text { if } & 1 \leq k_{N_{i}} \leq 1+E_{a_{i}, b_{i}} \\
M_{a_{i}, n}-p_{a_{i}}-1 & \text { if } & 1+E_{a_{i}, b_{i}}<k_{N_{i}} \leq 1+E_{a_{i}, b_{i}}+a_{i} \\
M_{a_{i}, n}-p_{a_{i}}-2 & \text { if } & 1+E_{a_{i}, b_{i}}+a_{i}<k_{N_{i}} \leq 1+E_{a_{i}, b_{i}}+2 a_{i} \\
\vdots & \vdots & \vdots \\
M_{a_{i}, n}-p_{a_{i}}-\widetilde{k}_{N_{i}} & \text { if } & 1+E_{a_{i}, b_{i}}+a_{i}\left(\widetilde{k}_{N_{i}}-1\right)<k_{N_{i}} \leq 1+E_{a_{i}, b_{i}}+a_{i} \tilde{k}_{N_{i}} \\
\vdots & \vdots & \vdots \\
M_{a_{i}, n}-p_{a_{i}}-\widetilde{k}_{N_{i}, \text { last }} & \text { if } & 1+E_{a_{i}, b_{i}}+a_{i}\left(\widetilde{k}_{N_{i}, \text { last }}-1\right)<k_{N_{i}} \leq p^{n}-N_{i, \max } .
\end{array}
$$

Proof. Observe that since $p^{n}-1=a_{i} M_{a_{i}, n}+r_{a_{i}, n}$, we have $p^{n}-k_{N_{i}}-a_{i} M-b_{i} \geq 0$ if and only if $M \leq M_{a_{i}, n}-\left(\left(b_{i}-r_{a_{i}, n}\right) / a_{i}\right)-$ $\left(\left(k_{N_{i}}-1\right) / a_{i}\right)$. So we need to compute the greatest positive integer $M$, that is, $\leq M_{a_{i}, n}-\left(\left(b_{i}-r_{a_{i}, n}\right) / a_{i}\right)-\left(\left(k_{N_{i}}-1\right) / a_{i}\right)$.

Case 1 (when $b_{i} \geq a_{i}$ ). In this case, we have $0 \leq r_{a_{i}, n}<a_{i} \leq b_{i}$. The least positive integer $\geq b_{i}-r_{a_{i}, n}$ which is divisible by $a_{i}$ is $y_{a_{i}}$. Therefore the least positive integer $\geq\left(b_{i}-r_{a_{i}, n}\right) / a_{i}$ is $q_{a_{i}}=$ $p_{a_{i}}$. Therefore the greatest negative integer $\leq-\left(b_{i}-r_{a_{i}, n}\right) / a_{i}$ is $-p_{a_{i}}$. And hence the greatest positive integer $\leq M_{a_{i}, n}-\left(b_{i}-\right.$ $\left.r_{a_{i}, n}\right) / a_{i}$ is $M_{a_{i}, n}-p_{a_{i}}$.

Since $k_{N_{i}} \leq p^{n}-N_{i, \max }$, it follows that $M_{a_{i}, n}-\left(\left(b_{i}-\right.\right.$ $\left.\left.r_{a_{i}, n}\right) / a_{i}\right)-\left(\left(k_{N_{i}}-1\right) / a_{i}\right) \geq M_{a_{i}, n}-p_{a_{i}}-\left(\left(p^{n}-N_{i, \max }-\right.\right.$
$\left.\left.1-E_{a_{i}, b_{i}}\right) / a_{i}\right)$. The smallest positive integer $\geq\left(p^{n}-N_{i, \max }-\right.$ $\left.1-E_{a_{i}, b_{i}}\right) / a_{i}$ is $\widetilde{k}_{N_{i} \text {, last }}$. Therefore the greatest positive integer $\leq M_{a_{i}, n}-p_{a_{i}}-\left(\left(p^{n}-N_{i, \max }-1-E_{a_{i}, b_{i}}\right) / a_{i}\right)$ is $M_{a_{i}, n}-p_{a_{i}}-\widetilde{k}_{N_{i} \text {, last }}$. As $k_{N_{i}}$ increases, $M_{a_{i}, n}-\left(\left(b_{i}-r_{a_{i}, n}\right) / a_{i}\right)-\left(\left(k_{N_{i}}-1\right) / a_{i}\right)$ decreases, the lower bound being equal to $M_{a_{i}, n}-p_{a_{i}}-\left(\left(p^{n}-\right.\right.$ $\left.\left.N_{i, \text { max }}-1-E_{a_{i}, b_{i}}\right) / a_{i}\right)$. Therefore the greatest positive integer $\leq M_{a_{i}, n}-\left(\left(b_{i}-r_{a_{i}, n}\right) / a_{i}\right)-\left(\left(k_{N_{i}}-1\right) / a_{i}\right)$ equals $M_{a_{i}, n}-p_{a_{i}}-\tilde{k}_{N_{i} \text { last }}$. Hence the greatest positive integer $\leq C_{i, n}:=M_{a_{i}, n}-\left(\left(b_{i}-\right.\right.$ $\left.\left.r_{a_{i}, n}\right) / a_{i}\right)-\left(\left(k_{N_{i}}-1\right) / a_{i}\right)$ is equal to

$$
\begin{array}{ccc}
M_{a_{i}, n}-p_{a_{i}} & \text { if } & M_{a_{i}, n}-p_{a_{i}} \leq C_{i, n} \\
M_{a_{i}, n}-p_{a_{i}}-1 & \text { if } & M_{a_{i}, n}-p_{a_{i}}-1 \leq C_{i, n}<M_{a_{i}, n}-p_{a_{i}} \\
M_{a_{i}, n}-p_{a_{i}}-2 & \text { if } & M_{a_{i}, n}-p_{a_{i}}-2 \leq C_{i, n}<M_{a_{i}, n}-p_{a_{i}}-1 \\
\vdots & \vdots & \vdots \\
M_{a_{i}, n}-p_{a_{i}}-\tilde{k}_{N_{i}, \text { last }} & \text { if } & M_{a_{i}, n}-p_{a_{i}}-\tilde{k}_{N_{i}, \text { last }} \leq C_{i, n}<M_{a_{i}, n}-p_{a_{i}}-\tilde{k}_{N_{i}, \text { last }}+1 .
\end{array}
$$

This is equivalent to saying that the maximum value of the positive integer $M$ for which $p^{n}-k_{N_{i}}-a_{i} M-b_{i}$ is $\geq 0$ equals

$$
\begin{array}{ccc}
M_{a_{i}, n}-p_{a_{i}} & \text { if } & 1 \leq k_{N_{i}} \leq 1+E_{a_{i}, b_{i}} \\
M_{a_{i}, n}-p_{a_{i}}-1 & \text { if } & 1+E_{a_{i}, b_{i}}<k_{N_{i}} \leq 1+E_{a_{i}, b_{i}}+a_{i} \\
M_{a_{i}, n}-p_{a_{i}}-2 & \text { if } & 1+E_{a_{i}, b_{i}}+a_{i}<k_{N_{i}} \leq 1+E_{a_{i}, b_{i}}+2 a_{i} \\
\vdots & \vdots & \vdots \\
M_{a_{i}, n}-p_{a_{i}}-\widetilde{k}_{N_{i}} & \text { if } & 1+E_{a_{i}, b_{i}}+a_{i}\left(\widetilde{k}_{N_{i}}-1\right)<k_{N_{i}} \leq 1+E_{a_{i}, b_{i}}+a_{i} \widetilde{k}_{N_{i}} \\
\vdots & \vdots & \vdots \\
M_{a_{i}, n}-p_{a_{i}}-\widetilde{k}_{N_{i}, \text { last }} & \text { if } & 1+E_{a_{i}, b_{i}}+a_{i}\left(\widetilde{k}_{N_{i}, \text { last }}-1\right)<k_{N_{i}} \leq p^{n}-N_{i, \max } .
\end{array}
$$


Case 2 (when $b_{i}<a_{i}$ ). In this case, we have 2 subcases.

Subcase 2.1 (when $b_{i} \leq r_{a_{i}, n}$ ). Here we have $p_{a_{i}}=0$ and $0 \leq$ $b_{i} \leq r_{a_{i}, n}<a_{i}$. Therefore $0 \leq\left(r_{a_{i}, n}-b_{i}\right) / a_{i}<1$ and hence $-1<\left(b_{i}-r_{a_{i}, n}\right) / a_{i} \leq 0$. Therefore the least nonnegative integer $\geq\left(b_{i}-r_{a_{i}, n}\right) / a_{i}$ is $p_{a_{i}}=0$. The rest of the proof is similar to the proof of Case 1 .

Subcase 2.2 (when $b_{i}>r_{a_{i}, n}$ ). Here we have, $p_{a_{i}}=1$ and $0 \leq$ $r_{a_{i}, n}<b_{i}<a_{i}$. Therefore $0<\left(b_{i}-r_{a_{i}, n}\right) / a_{i}<1$. Hence the least positive integer $\geq\left(b_{i}-r_{a_{i}, n}\right) / a_{i}$ is $p_{a_{i}}=1$. The rest of the proof is similar to the proof of Case 1 .

The following corollary is immediate.

Corollary 15. Let $A$ be a monomial in $\mathfrak{M}$ which [2] divides and let $M$ be a positive integer. Let $A$ and $A[2]^{-M}[1]^{M-1}$ be of the form as given in Remark 13. For $1 \leq i \leq r$, let $M_{i, A}$ be the maximum value of the positive integer $M$ for which $p^{n}-k_{N_{i}}-$ $a_{i} M-b_{i}$ is $\geq 0$.

Then $M_{\max , A}$ equals the minimum of all $M_{i, A} s$ as $i$ ranges from 1 to $r$.

Let $\mathfrak{M}[2]:=$ the set of all monomials in $\mathfrak{M}$ which are divisible by [2]. Let $M_{\max }[2]:=\left\{M_{\max , A} \mid A \in \mathfrak{M}[2]\right\}$.

Remark 16. It follows from Corollary 15 above that $M_{\max }[2]=$ $\left\{M_{a_{r}, n}-p_{a_{r}}-j \mid 0 \leq j \leq \widetilde{k}_{N_{r}, \text { last }}\right\}$.

\subsubsection{The Length Count for a Fixed $M_{\max , A}$}

Remark 17. It is easy to verify that if $M_{1}$ is a positive integer such that $A[2]^{-M_{1}}[1]^{M_{1}-1}$ contains no negative powers and $M_{2}$ is a positive integer such that $M_{2} \leq M_{1}$, then $A[2]^{-M_{2}}[1]^{M_{2}-1}$ contains no negative powers. It is also easy to verify that if $N_{1}$ is a positive integer such that $A[2]^{-N_{1}}[1]^{N_{1}}$ belongs to $A_{c}$ and $N_{2}$ is a positive integer such that $N_{1} \leq$ $N_{2}$, then $A[2]^{-N_{2}}[1]^{N_{2}}$ belongs to $A_{c}$. Hence for any $A \in$ $\mathfrak{M}[2], A \in A_{c}+J$ if and only if either [1] divides $A$ or $A[2]^{-M_{\max , A}}[1]^{M_{\max , A}}$ belongs to $A_{c}$.

Remark 18. Recall from Remark 13 as well as from Corollary 15 that given any monomial $A$ in $\mathfrak{M}[2]$, the value of $M_{\max , A}$ depends only on the powers of the negative difference variables appearing in $A$. It now follows that for each $i \in\{1, \ldots, r\}$, if we fix numbers $k_{N_{i}}^{0}$ such that $p^{n}-1 \geq p^{n}-k_{N_{i}}^{0} \geq N_{i, \max }$, then for any $A$ in the set

$$
\begin{aligned}
& \mathfrak{P}(\left.k_{N_{1}}^{0}, k_{N_{2}}^{0}, \ldots, k_{N_{r}}^{0}\right) \\
&:=\left\{A \mid A=N_{r}^{p^{n}-k_{N_{r}}^{0}} \cdots N_{1}^{p^{n}-k_{N_{1}}^{0}} Z_{s}^{l_{s}} \cdots Z_{1}^{l_{1}} P_{1}^{j_{1}} \cdots P_{t}^{j_{t}}\right. \\
& \text { where } p^{n}-1 \geq l_{j} \geq 0
\end{aligned}
$$

for every $j \in\{1, \ldots, s\}$ and,

$$
\left.p^{n}-1 \geq j_{q} \geq 0 \text { for every } q \in\{1, \ldots, t\}\right\},
$$

the value of $M_{\max , A}$ is the same (call it $M_{\max }^{0}$ ).
The main result of this subsubsection is Lemma 24 below which provides an account of the length count for a fixed value of $M_{\max , A}$. But for stating the lemma, we need to introduce some notation first.

For an arbitrary element $M_{\max }$ in $M_{\max }$ [2] and for every $q \in\{1, \ldots, t\}$, let

$$
\operatorname{Min}_{P_{q}, M_{\max }}^{c}:=\operatorname{Min}\left\{p^{n}-P_{q, \min }, M_{\text {max }} \Delta_{P_{q}}\right\} .
$$

The symbol $\mathrm{Min}^{c}$ in the above definition of $\operatorname{Min}_{P_{q}, M_{\max }}^{c}$ stands for "minimum convergent." The reason behind this notation is explained in Remark 19 below.

Remark 19. Observe that if the power of the variable $P_{q}$ in the monomial $A$ is $j_{q}$, then the power of $P_{q}$ in the monomial $A[2]^{-M_{\max }[1]^{M_{\max }}}$ is $j_{q}+M_{\max } \Delta_{P_{q}}$. If $A$ is divisible by [2], then $p^{n}-1 \geq j_{q} \geq P_{q, \min }$. Now if $j_{q}+M_{\max } \Delta_{P_{q}} \geq p^{n}$, then $A[2]^{-M_{\max }}[1]^{M_{\max }}$ belongs to $A_{c}$. That is, if $p^{n}-j_{q} \leq$ $\operatorname{Min}_{P_{q}, M_{\max }}^{c}$, then $A[2]^{-M_{\max }}[1]^{M_{\max }}$ belongs to $A_{c}$. That is, if $p^{n}-\operatorname{Min}_{P_{q}, M_{\max }}^{c} \leq j_{q} \leq p^{n}-1$, then $A[2]^{-M_{\max }}[1]^{M_{\max }}$ belongs to $A_{c}$. So $p^{n}-\operatorname{Min}_{P_{q}, M_{\max }}^{c}$ is the minimum possible power of the variable $P_{q}$ in the monomial $A$ for which $A[2]^{-M_{\max }}[1]^{M_{\max }}$ belongs to $A_{c}$.

Recall the ordered set $P_{1} \triangleright \cdots \triangleright P_{t}$ of positive difference variables. Let $A_{t+1}:=0$ and $C_{t}:=1$. For any $q \in\{1, \ldots, t-1\}$, let

$$
C_{q}:=p^{(t-q) n}
$$

And for any $q \in\{2, \ldots, t\}$, let

$$
A_{q}:=\left(p^{n}-P_{q, \min }\right) A_{q+1}+P_{q, \min } C_{q} .
$$

For any $M_{\max }$ in $M_{\max }[2]$, let $D_{t+1, M_{\max }}:=1$ and $\widetilde{D}_{t+1, M_{\max }}:=$ 0 . For any $M_{\max }$ in $M_{\max }[2]$ and any $q \in\{1, \ldots, t\}$, let

$$
\begin{aligned}
& D_{q, M_{\text {max }}}:=\left(\operatorname{Min}_{P_{q}, M_{\text {max }}}^{c}\right) A_{q+1} \\
& +\left(p^{n}-P_{q, \min }-\operatorname{Min}_{P_{q}, M_{\max }}^{c}\right) D_{q+1, M_{\text {max }}} \\
& +\left(P_{q, \min }\right) C_{q}, \\
& \left\{\begin{array}{l}
\left(\operatorname{Min}_{P_{q}, M_{\text {max }}}^{c}\right) A_{q+1} \\
\quad+\left(p^{n}-\operatorname{Min}_{P_{q}, M_{\max }}^{c}-P_{q, \max }\right) \widetilde{D}_{q+1, M_{\max }}
\end{array}\right. \\
& +\left(P_{q, \max }-P_{q, \min }\right) D_{q+1, M_{\max }}+\left(P_{q, \min }\right) C_{q} \\
& \text { if } \operatorname{Min}_{P_{q}, M_{\max }}^{c}<p^{n}-P_{q, \min }
\end{aligned}
$$

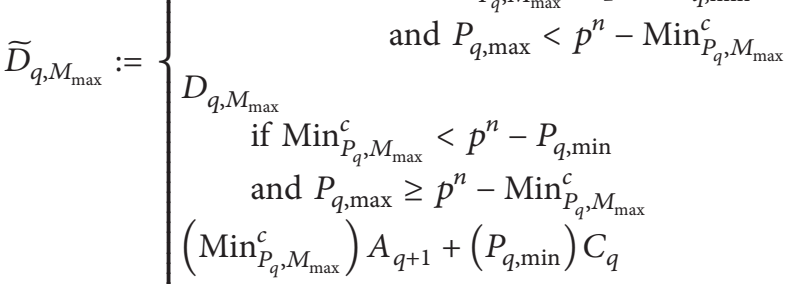

$$
\begin{aligned}
& \text { if } \operatorname{Min}_{P_{q}, M_{\max }}^{c}=p^{n}-P_{q, \min } \text {. }
\end{aligned}
$$


Recall the set $\mathfrak{P}\left(k_{N_{1}}^{0}, k_{N_{2}}^{0}, \ldots, k_{N_{r}}^{0}\right)$ from Remark 18. For any $A$ in this set $\mathfrak{P}\left(k_{N_{1}}^{0}, k_{N_{2}}^{0}, \ldots, k_{N_{r}}^{0}\right)$, the value of $M_{\max , A}$ is the same, namely, $M_{\text {max }}^{0}$. Lemma 24 below gives an account of the length count for the set $\mathfrak{P}\left(k_{N_{1}}^{0}, k_{N_{2}}^{0}, \ldots, k_{N_{r}}^{0}\right)$. But we will break up the proof of Lemma 24 in a few short steps as follows.

Lemma 20. For every $q \in\{2, \ldots, t\}$, let

$$
\begin{aligned}
\mathfrak{\mho}_{q, A, J_{1}, \ldots, J_{q-1}} & \\
:=\{A & =N_{r}^{p^{n}-k_{N_{r}}^{0}} \cdots N_{1}^{p^{n}-k_{N_{1}}^{0}} Z_{s}^{l_{s}} \cdots Z_{1}^{l_{1}} P_{1}^{j_{1}} \cdots P_{t}^{j_{t}} \\
& \in \mathfrak{P}\left(k_{N_{1}}^{0}, k_{N_{2}}^{0}, \ldots, k_{N_{r}}^{0}\right) \mid l_{s}=l_{s-1}=\cdots \\
& \left.=l_{1}=p^{n}-1 \text { and } j_{1}=J_{1}, \ldots, j_{q-1}=J_{q-1}\right\},
\end{aligned}
$$

where for each $k \in\{1, \ldots, q-1\}, J_{k}$ is a fixed integer such that $P_{k, \min } \leq J_{k} \leq p^{n}-1$ and at least one $J_{k}$ is $\geq p^{n}-\operatorname{Min}_{P_{k}, M_{\max }^{0}}$.

Then the total number of monomials $A$ in the set $\mathfrak{\mho}_{q, A, J_{1}, \ldots, J_{q-1}}$ for which $A \notin A_{c}+J$ equals $A_{q}$.

Proof. The proof is by reverse induction on $q$, the base case of induction being $q=t$. Observe that if $A$ is any monomial in $\mathfrak{\mho}_{t, A, J_{1}, \ldots, J_{t-1}}$ such that the power $j_{t}$ of the variable $P_{t}$ in it satisfies the inequality $P_{t, \text { min }} \leq j_{t} \leq p^{n}-1$, then [2] divides

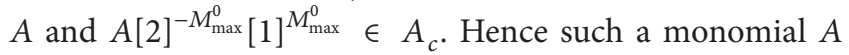
satisfies $A \in A_{c}+J$. The total number of such monomials in $\mathfrak{\mho}_{t, A, J_{1}, \ldots, J_{t-1}}$ is $p^{n}-P_{t, \min }$. And if $A$ is any monomial in $\mathfrak{F}_{t, A, J_{1}, \ldots, J_{t-1}}$ such that the power $j_{t}$ of the variable $P_{t}$ in it satisfies the inequality $0 \leq j_{t}<P_{t, \min }$, then neither [2] nor [1] divides $A$ and hence $A \notin A_{c}+J$. The total number of such monomials in $\mathfrak{F}_{t, A, J_{1}, \ldots, J_{t-1}}$ is $P_{t, \text { min }}$. Hence the total number of monomials $A$ in the set $\mathfrak{F}_{t, A, J_{1}, \ldots, J_{t-1}}$ for which $A \notin A_{c}+J$ equals

$$
\begin{aligned}
\left(p^{n}-P_{t, \text { min }}\right) 0+\left(P_{t, \text { min }}\right) 1= & \left(p^{n}-P_{t, \text { min }}\right) A_{t+1} \\
& +\left(P_{t, \text { min }}\right) C_{t}=A_{t} .
\end{aligned}
$$

This proves the base case of induction. The rest of the proof follows by reverse induction and is left to the reader.

Lemma 21. For every $q \in\{2, \ldots, t\}$, let

$$
\begin{aligned}
& \mathfrak{F}_{q, C, J_{1}, \ldots, J_{q-1}} \\
& :=\left\{A=N_{r}^{p^{n}-k_{N_{r}}^{0}} \cdots N_{1}^{p^{n}-k_{N_{1}}^{0}} Z_{s}^{l_{s}} \cdots Z_{1}^{l_{1}} P_{1}^{j_{1}} \cdots P_{t}^{j_{t}}\right. \\
& \in \mathfrak{P}\left(k_{N_{1}}^{0}, k_{N_{2}}^{0}, \ldots, k_{N_{r}}^{0}\right) \mid l_{s}=l_{s-1}=\cdots \\
& \left.=l_{1}=p^{n}-1, j_{1}=J_{1}, \ldots, j_{q-1}=J_{q-1}\right\},
\end{aligned}
$$

where for each $k \in\{1, \ldots, q-1\}, J_{k}$ is a fixed integer such that $0 \leq J_{k} \leq p^{n}-1$ and at least one $J_{k}$ is $<P_{k, \min }$.
Then the total number of monomials $A$ in the set $\mathfrak{\mho}_{q, C, J_{1}, \ldots, J_{q-1}}$ for which $A \notin A_{c}+J$ equals $C_{q-1}$.

Proof. The proof is by reverse induction on $q$, the base case of induction being $q=t$. Observe that if $A$ is any monomial in $\mathfrak{F}_{t, C, J_{1}, \ldots, J_{t-1}}$ such that the power $j_{t}$ of the variable $P_{t}$ in it satisfies the inequality $0 \leq j_{t} \leq p^{n}-1$, then neither [2] nor [1] divides $A$ and hence $A \notin A_{c}+J$. The total number of such monomials in $\mathfrak{\mho}_{t, C, J_{1}, \ldots, J_{t-1}}$ is $p^{n}$. Hence the total number of monomials $A$ in the set $\mathfrak{\mho}_{t, C, J_{1}, \ldots, J_{t-1}}$ for which $A \notin A_{c}+J$ equals $p^{n}=p^{(t-(t-1)) n}=C_{t-1}$. This proves the base case of induction. The rest of the proof follows by reverse induction and is left to the reader.

Lemma 22. For every $q \in\{2, \ldots, t\}$, let

$$
\begin{aligned}
& \mathfrak{\mho}_{q, D, J_{1}, \ldots, J_{q-1}} \\
& :=\left\{A=N_{r}^{p^{n}-k_{N_{r}}^{0}} \cdots N_{1}^{p^{n}-k_{N_{1}}^{0}} Z_{s}^{l_{s}} \cdots Z_{1}^{l_{1}} P_{1}^{j_{1}} \cdots P_{t}^{j_{t}}\right. \\
& \in \mathfrak{P}\left(k_{N_{1}}^{0}, k_{N_{2}}^{0}, \ldots, k_{N_{r}}^{0}\right) \mid l_{s}=l_{s-1}=\cdots \\
& \left.=l_{1}=p^{n}-1 \text { and } j_{1}=J_{1}, \ldots, j_{q-1}=J_{q-1}\right\} \text {, }
\end{aligned}
$$

where for each $k \in\{1, \ldots, q-1\}, J_{k}$ is a fixed integer such that $P_{k, \text { min }} \leq J_{k}<p^{n}-\operatorname{Min}_{P_{k}, M_{\max }^{0}}^{c}$.

Then the total number of monomials $A$ in the set $\mathfrak{F}_{q, D, J_{1}, \ldots, J_{q-1}}$ for which $A \notin A_{c}+J$ equals

(i) $D_{q, M_{\max }^{0}}$ if there exists at least one $k \in\{1, \ldots, q-1\}$ such that $P_{k, \max } \geq p^{n}-\operatorname{Min}_{P_{k}, M_{\max }^{0}}^{c}$;

(ii) $\widetilde{D}_{q, M_{\max }^{0}}$ iffor every $k \in\{1, \ldots, q-1\}$, we have $P_{k, \max }<$ $p^{n}-\operatorname{Min}_{P_{k}, M_{\max }^{0}}^{c}$ and $P_{k, \max } \leq J_{k}<p^{n}-\operatorname{Min}_{P_{k}, M_{\max }^{0}}^{c}$;

(iii) $D_{q, M_{\max }^{0}}$ if for every $k \in\{1, \ldots, q-1\}$, we have $P_{k, \max }<$ $p^{n}-\operatorname{Min}_{P_{k}, M_{\max }^{0}}^{c}$ and there exists at least one $k \in$ $\{1, \ldots, q-1\}$ such that $P_{k, \min } \leq J_{k}<P_{k, \max }$.

Proof. The proof is by reverse induction on $q$, the base case of induction being $q=t$. Let us first prove the base case of induction.

Case 1. When there exists at least one $k \in\{1, \ldots, t-1\}$ such that $P_{k, \text { max }} \geq p^{n}-\operatorname{Min}_{P_{k}, M_{\max }^{0}}^{c}$.

Then since we have that for each $k \in\{1, \ldots, t-1\}, J_{k}$ is a fixed integer such that $P_{k, \min } \leq J_{k}<p^{n}-\operatorname{Min}_{P_{k}, M_{\max }^{0}}^{c}$, it follows that monomials in the set $\mathfrak{F}_{t, D, J_{1}, \ldots, J_{t-1}}$ are not divisible by [1].

Observe now that if $A$ is any monomial in $\mathfrak{\mho}_{t, D, J_{1}, \ldots, J_{t-1}}$ such that the power $j_{t}$ of the variable $P_{t}$ in it satisfies the inequality $p^{n}-\operatorname{Min}_{P_{t}, M_{\max }^{0}}^{c} \leq j_{t} \leq p^{n}-1$, then [2] divides

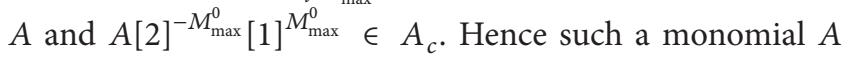
satisfies $A \in A_{c}+J$. The total number of such monomials in $\mathfrak{\mho}_{t, D, J_{1}, \ldots, J_{t-1}}$ is $\operatorname{Min}_{P_{t}, M_{\max }^{0}}^{c}$. If $A$ is any monomial in $\mathfrak{\mho}_{t, D, J_{1}, \ldots, J_{t-1}}$ such that the power $j_{t}$ of the variable $P_{t}$ in it satisfies the inequality $P_{t, \text { min }} \leq j_{t}<p^{n}-\operatorname{Min}_{P_{t}, M_{\text {max }}^{0}}^{c}$, then [2] divides $A$ but [1] does not divide $A$ and $A[2]^{-M_{\max }^{0}}[1]^{M_{\max }^{0}} \notin A_{c}$. Hence 
such a monomial $A$ satisfies $A \notin A_{c}+J$. The total number of such monomials in $\mathfrak{\Im}_{t, D, J_{1}, \ldots, J_{t-1}}$ is $p^{n}-\operatorname{Min}_{P_{t}, M_{\max }^{0}}^{c}-P_{t, \min }$. And if $A$ is any monomial in $\mathfrak{F}_{t, D, J_{1}, \ldots, J_{t-1}}$ such that the power $j_{t}$ of the variable $P_{t}$ in it satisfies the inequality $0 \leq j_{t}<P_{t \text {,min }}$, then neither [2] nor [1] divides $A$ and hence $A \notin A_{c}+J$. The total number of such monomials in $\mathfrak{F}_{t, D, J_{1}, \ldots, J_{t-1}}$ is $P_{t, \min }$. Hence the total number of monomials $A$ in the set $\mathfrak{\Im}_{t, D, J_{1}, \ldots, J_{t-1}}$ for which $A \notin A_{c}+J$ equals

$$
\begin{aligned}
& \left(\operatorname{Min}_{P_{t}, M_{\max }^{0}}^{c}\right) 0+\left(p^{n}-\operatorname{Min}_{P_{t}, M_{\max }^{0}}^{c}-P_{t, \min }\right) 1+\left(P_{t, \min }\right) 1 \\
& =\left(\operatorname{Min}_{P_{t}, M_{\max }^{0}}^{c}\right) A_{t+1}+\left(p^{n}-\operatorname{Min}_{P_{t}, M_{\max }^{0}}^{c}-P_{t, \min }\right) D_{t+1, M_{\max }^{0}} \\
& \quad+\left(P_{t, \min }\right) C_{t}=D_{t, M_{\max }^{0}} .
\end{aligned}
$$

This proves the base case of induction in Case 1.

Case 2. When for every $k \in\{1, \ldots, t-1\}$, we have $P_{k \text {, max }}<$ $p^{n}-\operatorname{Min}_{P_{k}, M_{\max }^{0}}^{c}$ and $P_{k, \text { max }} \leq J_{k}<p^{n}-\operatorname{Min}_{P_{k}, M_{\max }^{0}}^{c}$. In this case, there are 3 subcases.

Subcase $2.1\left(\operatorname{Min}_{P_{t}, M_{\max }^{0}}^{c}=p^{n}-P_{t, \min }\right)$. Observe that if $A$ is any monomial in $\mathfrak{\mho}_{t, D, J_{1}, \ldots, J_{t-1}}$ such that the power $j_{t}$ of the variable $P_{t}$ in it satisfies the inequality $p^{n}-\operatorname{Min}_{P_{t}, M_{\max }^{0}}^{c} \leq j_{t} \leq$ $p^{n}-1$, then [2] divides $A$ and $A[2]^{-M_{\max }^{0}}[1]^{M_{\max }^{0}} \in A_{c}$. Hence such a monomial $A$ satisfies $A \in A_{c}+J$. The total number of such monomials in $\mathfrak{F}_{t, D, J_{1}, \ldots, J_{t-1}}$ is $\operatorname{Min}_{P_{t}, M_{\max }^{0}}^{c}$. And if $A$ is any monomial in $\mathfrak{\Im}_{t, D, J_{1}, \ldots, J_{t-1}}$ such that the power $j_{t}$ of the variable $P_{t}$ in it satisfies the inequality $0 \leq j_{t}<P_{t, \min }=p^{n}-\operatorname{Min}_{P_{t}, M_{\max }^{0}}^{c}$, then neither [2] nor [1] divides $A$ and hence $A \notin A_{c}+J$. The total number of such monomials in $\mathfrak{\Im}_{t, D, J_{1}, \ldots, J_{t-1}}$ is $P_{t, \text { min }}$. Hence the total number of monomials $A$ in the set $\mathfrak{\Im}_{t, D, J_{1}, \ldots, J_{t-1}}$ for which $A \notin A_{c}+J$ equals

$$
\begin{aligned}
& \left(\operatorname{Min}_{P_{t}, M_{\max }^{0}}^{c}\right) 0+\left(P_{t, \min }\right) 1 \\
& \quad=\left(\operatorname{Min}_{P_{t}, M_{\max }^{0}}\right) A_{t+1}+\left(P_{t, \min }\right) C_{t}=\widetilde{D}_{t, M_{\max }^{0}} .
\end{aligned}
$$

This proves the base case of induction in this subcase.

Subcase $2.2\left(\operatorname{Min}_{P_{t}, M_{\max }^{0}}^{c}<p^{n}-P_{t, \text { min }}\right.$ and $P_{t, \max } \geq p^{n}-$ $\left.\operatorname{Min}_{P_{t}, M_{\max }^{0}}^{c}\right)$. Observe that if $A$ is any monomial in $\mathfrak{\Im}_{t, D, J_{1}, \ldots, J_{t-1}}$ such that the power $j_{t}$ of the variable $P_{t}$ in it satisfies the inequality $p^{n}-\operatorname{Min}_{P_{t}, M_{\max }^{0}}^{c} \leq j_{t} \leq p^{n}-1$, then [2] divides $A$ and $A[2]^{-M_{\max }^{0}}[1]^{M_{\max }^{0}} \in A_{c}$. Hence such a monomial $A$ satisfies $A \in A_{c}+J$. The total number of such monomials in $\mathfrak{\mho}_{t, D, J_{1}, \ldots, J_{t-1}}$ is $\operatorname{Min}_{P_{t}, M_{\max }^{0}}^{c}$. If $A$ is any monomial in $\mathfrak{\mho}_{t, D, J_{1}, \ldots, J_{t-1}}$ such that the power $j_{t}$ of the variable $P_{t}$ in it satisfies the inequality $P_{t, \text { min }} \leq j_{t}<p^{n}-\operatorname{Min}_{P_{t}, M_{\max }^{0}}^{c}$, then [2] divides $A$ but [1] does not divide $A$ (since $P_{t, \max } \geq p^{n}-\operatorname{Min}_{P_{t}, M_{\max }^{0}}^{c}$ ) and $A[2]^{-M_{\max }^{0}}[1]^{M_{\max }^{0}} \notin A_{c}$. Hence such a monomial $A$ satisfies $A \notin A_{c}+J$. The total number of such monomials in $\mathfrak{\Im}_{t, D, J_{1}, \ldots, J_{t-1}}$ is $p^{n}-\operatorname{Min}_{P_{t}, M_{\max }^{0}}^{c}-P_{t, \min }$. And if $A$ is any monomial in $\mathfrak{F}_{t, D, J_{1}, \ldots, J_{t-1}}$ such that the power $j_{t}$ of the variable $P_{t}$ in it satisfies the inequality $0 \leq j_{t}<P_{t \text {,min }}$, then neither [2] nor [1] divides $A$ and hence $A \notin A_{c}+J$. The total number of such monomials in $\mathfrak{\mho}_{t, D, J_{1}, \ldots, J_{t-1}}$ is $P_{t, \text { min }}$. Hence the total number of monomials $A$ in the set $\mathfrak{\Im}_{t, D, J_{1}, \ldots, J_{t-1}}$ for which $A \notin A_{c}+J$ equals

$$
\begin{aligned}
& \left(\operatorname{Min}_{P_{t}, M_{\max }^{0}}^{c}\right) 0+\left(p^{n}-\operatorname{Min}_{P_{t}, M_{\max }^{0}}^{c}-P_{t, \min }\right) 1+\left(P_{t, \min }\right) 1 \\
& =\left(\operatorname{Min}_{P_{t}, M_{\max }^{0}}^{c}\right) A_{t+1}+\left(p^{n}-\operatorname{Min}_{P_{t}, M_{\max }^{0}}^{c}-P_{t, \min }\right) D_{t+1, M_{\max }^{0}} \\
& \quad+\left(P_{t, \min }\right) C_{t}=D_{t, M_{\max }^{0}}=\widetilde{D}_{t, M_{\max }^{0}} .
\end{aligned}
$$

This proves the base case of induction in this subcase.

Subcase $2.3\left(\operatorname{Min}_{P_{t}, M_{\max }^{0}}^{c}<p^{n}-P_{t, \min }\right.$ and $P_{t, \max }<p^{n}-$ $\left.\operatorname{Min}_{P_{t}, M_{\max }^{0}}^{c}\right)$. Observe that if $A$ is any monomial in $\mathfrak{\Im}_{t, D, J_{1}, \ldots, J_{t-1}}$ such that the power $j_{t}$ of the variable $P_{t}$ in it satisfies the inequality $p^{n}-\operatorname{Min}_{P_{t}, M_{\max }^{0}}^{c} \leq j_{t} \leq p^{n}-1$, then [2] divides $A$ and $A[2]^{-M_{\max }^{0}}[1]^{M_{\max }^{0}} \in A_{c}$. Hence such a monomial $A$ satisfies $A \in A_{c}+J$. The total number of such monomials in $\mathfrak{F}_{t, D, J_{1}, \ldots, J_{t-1}}$ is $\operatorname{Min}_{P_{t}, M_{\max }^{0}}^{c}$. If $A$ is any monomial in $\mathfrak{\Im}_{t, D, J_{1}, \ldots, J_{t-1}}$ such that the power $j_{t}$ of the variable $P_{t}$ in it satisfies the inequality $P_{t, \max } \leq j_{t}<p^{n}-\operatorname{Min}_{P_{t}, M_{\text {max }}^{0}}^{c}$, then [1] divides $A$ (since $P_{t, \text { max }} \leq j_{t}$ and $P_{k, \text { max }} \leq J_{k}<p^{n}-\operatorname{Min}_{P_{k}, M_{\max }^{0}}^{c}$ for every $k \in\{1, \ldots, t-1\})$. Hence such a monomial $A$ satisfies $A \in$ $A_{c}+J$. The total number of such monomials in $\mathfrak{\Im}_{t, D, J_{1}, \ldots, J_{t-1}}$ is $p^{n}-\operatorname{Min}_{P_{t}, M_{\max }^{0}}^{c}-P_{t, \max }$. If $A$ is any monomial in $\mathfrak{\Im}_{t, D, J_{1}, \ldots, J_{t-1}}$ such that the power $j_{t}$ of the variable $P_{t}$ in it satisfies the inequality $P_{t, \text { min }} \leq j_{t}<P_{t, \text { max }}$, then [2] divides $A$ but [1] does not divide $A$ (since $j_{t}<P_{t, \max }$ ) and $A[2]^{-M_{\max }^{0}[1]^{M_{\max }^{0}} \notin A_{c}}$ (since $j_{t}<P_{t, \max }<p^{n}-\operatorname{Min}_{P_{t}, M_{\max }^{0}}$ ). Hence such a monomial $A$ satisfies $A \notin A_{c}+J$. The total number of such monomials in $\mathfrak{F}_{t, D, J_{1}, \ldots, J_{t-1}}$ is $P_{t, \max }-P_{t, \min }$. And if $A$ is any monomial in $\mathfrak{F}_{t, D, J_{1}, \ldots, J_{t-1}}$ such that the power $j_{t}$ of the variable $P_{t}$ in it satisfies the inequality $0 \leq j_{t}<P_{t, \text { min }}$, then neither [2] nor [1] divides $A$ and hence $A \notin A_{c}+J$. The total number of such monomials in $\mathfrak{\Im}_{t, D, J_{1}, \ldots, J_{t-1}}$ is $P_{t, \text { min }}$. Hence the total number of monomials $A$ in the set $\mathfrak{\Im}_{t, D, J_{1}, \ldots, J_{t-1}}$ for which $A \notin A_{c}+J$ equals

$$
\begin{aligned}
\left(\operatorname{Min}_{P_{t}, M_{\max }^{0}}^{c}\right) 0+\left(p^{n}-\operatorname{Min}_{P_{t}, M_{\max }^{0}}^{c}-P_{t, \max }\right) 0 \\
\quad+\left(P_{t, \max }-P_{t, \min }\right) 1+\left(P_{t, \min }\right) 1 \\
=\left(\operatorname{Min}_{P_{t}, M_{\max }^{0}}\right) A_{t+1}+\left(p^{n}-\operatorname{Min}_{P_{t}, M_{\max }^{0}}-P_{t, \max }\right) \\
\quad \times \widetilde{D}_{t+1, M_{\max }^{0}}+\left(P_{t, \max }-P_{t, \min }\right) D_{t+1, M_{\max }^{0}}+\left(P_{t, \min }\right) C_{t} \\
=\widetilde{D}_{t, M_{\max }^{0}} .
\end{aligned}
$$

This proves the base case of induction in this subcase. Hence the base case of induction is proved in Case 2.

Case 3. When for every $k \in\{1, \ldots, t-1\}$, we have $P_{k \text {, max }}<$ $p^{n}-\operatorname{Min}_{P_{k}, M_{\max }^{0}}^{c}$ and there exists at least one $k \in\{1, \ldots, t-1\}$ such that $P_{k, \min } \leq J_{k}<P_{k, \text { max }}$.

Since there exists at least one $k \in\{1, \ldots, t-1\}$ such that $P_{k \text {, min }} \leq J_{k}<P_{k \text {, max }}$, it follows that monomials in the set 
$\mathfrak{F}_{t, D, J_{1}, \ldots, J_{t-1}}$ are not divisible by [1]. The rest of the proof of this case is similar to the proof of Case 1. This proves the base case of induction in Case 3.

This proves the base case of induction. The rest of the proof follows by reverse induction and is left to the reader.

$$
\begin{aligned}
& \text { Let } \\
& \qquad \begin{aligned}
\mathfrak{F}_{1}:=\{A & =N_{r}^{p^{n}-k_{N_{r}}^{0}} \cdots N_{1}^{p^{n}-k_{N_{1}}^{0}} Z_{s}^{l_{s}} \cdots Z_{1}^{l_{1}} P_{1}^{j_{1}} \cdots P_{t}^{j_{t}} \\
& \in \mathfrak{P}\left(k_{N_{1}}^{0}, k_{N_{2}}^{0}, \ldots, k_{N_{r}}^{0}\right) \mid l_{s}=l_{s-1}=\cdots \\
& \left.=l_{1}=p^{n}-1\right\} .
\end{aligned}
\end{aligned}
$$

Lemma 23. The total number of monomials $A$ in the set $\mathfrak{F}_{1}$ for which $A \notin A_{c}+J$ equals $\widetilde{D}_{1, M_{\max }^{0}}$.

Proof. Let $J_{1}$ be an arbitrarily fixed integer such that $p^{n}-$ $\operatorname{Min}_{P_{1}, M_{\max }^{0}}^{c} \leq J_{1} \leq p^{n}-1$. It then follows from Lemma 20 that for any such fixed $J_{1}$, the total number of monomials $A$ in $\mathfrak{F}_{2, A, J}$ such that $A \notin A_{c}+J$ equals $A_{2}$. Also if $J_{1}$ is an arbitrarily fixed integer such that $0 \leq J_{1}<P_{1, \min }$, then it follows from Lemma 21 that for any such fixed $J_{1}$, the total number of monomials $A$ in $\mathfrak{\mho}_{2, C, J_{1}}$ such that $A \notin A_{c}+J$ equals $C_{1}$. Now there are 3 cases.

Case 1 (if $\operatorname{Min}_{P_{1}, M_{\max }^{0}}^{c}=p^{n}-P_{1, \min }$ ). In this case, the total number of monomials $A$ in the set $\mathfrak{F}_{1}$ for which $A \notin A_{c}+J$ equals

$$
\begin{gathered}
\left(p^{n}-1-p^{n}+\operatorname{Min}_{P_{1}, M_{\max }^{0}}^{c}+1\right) A_{2}+\left(P_{1, \min }\right) C_{1} \\
=\left(\operatorname{Min}_{P_{1}, M_{\max }^{0}}^{c}\right) A_{2}+\left(P_{1, \min }\right) C_{1}=\widetilde{D}_{1, M_{\max }^{0}} .
\end{gathered}
$$

Case 2 (if $\operatorname{Min}_{P_{1}, M_{\max }^{0}}^{c}<p^{n}-P_{1, \min }$ and $P_{1, \max } \geq p^{n}-$ $\operatorname{Min}_{P_{1}, M_{\max }^{0}}^{c}$. It follows from Lemma 22 that for any fixed integer $J_{1}$ such that $P_{1, \min } \leq J_{1}<p^{n}-\operatorname{Min}_{P_{1}, M_{\max }^{0}}^{c}$, the total number of monomials $A$ in $\mathfrak{\mho}_{2, D, J_{1}}$ for which $A \notin A_{c}+J$ equals $D_{2, M_{\max }^{0}}$. Hence in this case, the total number of monomials $A$ in the set $\mathfrak{\mho}_{1}$ for which $A \notin A_{c}+J$ equals

$$
\begin{aligned}
\left(p^{n}-\right. & \left.1-p^{n}+\operatorname{Min}_{P_{1}, M_{\max }^{0}}^{c}+1\right) A_{2} \\
& +\left(p^{n}-\operatorname{Min}_{P_{1}, M_{\max }^{0}}^{c}-P_{1, \min }\right) D_{2, M_{\max }^{0}}+\left(P_{1, \min }\right) C_{1} \\
= & \left(\operatorname{Min}_{P_{1}, M_{\max }^{0}}^{c}\right) A_{2}+\left(p^{n}-\operatorname{Min}_{P_{1}, M_{\max }^{0}}^{c}-P_{1, \min }\right) D_{2, M_{\max }^{0}} \\
& +\left(P_{1, \min }\right) C_{1}=D_{1, M_{\max }^{0}}=\widetilde{D}_{1, M_{\max }^{0}} .
\end{aligned}
$$

Case 3 (if $\operatorname{Min}_{P_{1}, M_{\max }^{0}}^{c}<p^{n}-P_{1, \min }$ and $P_{1, \max }<p^{n}-$ $\operatorname{Min}_{P_{1}, M_{\max }^{0}}^{c}$. It follows from Lemma 22 that for any fixed integer $J_{1}$ such that $P_{1, \max } \leq J_{1}<p^{n}-\operatorname{Min}_{P_{1}, M_{\max }^{0}}^{c}$, the total number of monomials $A$ in $\mathfrak{\mho}_{2, D, J_{1}}$ for which $A \notin A_{c}+J$ equals
$\widetilde{D}_{2, M_{\max }^{0}}$. It again follows from Lemma 22 that for any fixed integer $J_{1}$ such that $P_{1, \text { min }} \leq J_{1}<P_{1, \text { max }}$, the total number of monomials $A$ in $\mathfrak{\mho}_{2, D, J_{1}}$ for which $A \notin A_{c}+J$ equals $D_{2, M_{\max }^{0}}$. Hence in this case, the total number of monomials $A$ in the set $\mathfrak{F}_{1}$ for which $A \notin A_{c}+J$ equals

$$
\begin{aligned}
\left(p^{n}-\right. & \left.1-p^{n}+\operatorname{Min}_{P_{1}, M_{\max }^{0}}^{c}+1\right) A_{2} \\
& +\left(p^{n}-\operatorname{Min}_{P_{1}, M_{\max }^{0}}^{c}-P_{1, \max }\right) \widetilde{D}_{2, M_{\max }^{0}} \\
& +\left(P_{1, \max }-P_{1, \min }\right) D_{2, M_{\max }^{0}}+\left(P_{1, \min }\right) C_{1} \\
= & \left(\operatorname{Min}_{P_{1}, M_{\max }^{0}}^{c}\right) A_{2}+\left(p^{n}-\operatorname{Min}_{P_{1}, M_{\max }^{0}}^{c}-P_{1, \max }\right) \widetilde{D}_{2, M_{\max }^{0}} \\
& +\left(P_{1, \max }-P_{1, \min }\right) D_{2, M_{\max }^{0}}+\left(P_{1, \min }\right) C_{1}=\widetilde{D}_{1, M_{\max }^{0}} .
\end{aligned}
$$

Lemma 24. The total number of monomials $A$ in the set $\mathfrak{P}\left(k_{N_{1}}^{0}, k_{N_{2}}^{0}, \ldots, k_{N_{r}}^{0}\right)$ for which $A \notin A_{c}+J$ equals $\mathfrak{\Im}_{M_{\text {max }}^{0},}$ where $\mathfrak{S}_{M_{\max }^{0}, s}$ is defined inductively as follows:

$$
\begin{gathered}
\mathfrak{S}_{M_{\max }^{0}, 0}:=\widetilde{D}_{1, M_{\max }^{0}}, \quad \text { for every } j \in\{1, \ldots, s\}, \\
\mathfrak{S}_{M_{\max }^{0}, j}:=\left(\widetilde{S}_{M_{\max }^{0}, j-1}\right)\left(p^{n}-Z_{j, \text { min }}\right)+\left(p^{(t+j-1) n}\right)\left(Z_{j, \text { min }}\right) .
\end{gathered}
$$

Proof. Fix an arbitrary integer $L_{1}$ such that $p^{n}-1 \geq L_{1} \geq$ $Z_{1, \text { min }}$. Let

$$
\begin{aligned}
\mathfrak{P}_{L_{1}}:=\{A & =N_{r}^{p^{n}-k_{N_{r}}^{0}} \cdots N_{1}^{p^{n}-k_{N_{1}}^{0}} Z_{s}^{l_{s}} \cdots Z_{1}^{l_{1}} P_{1}^{j_{1}} \cdots P_{t}^{j_{t}} \\
& \in \mathfrak{P}\left(k_{N_{1}}^{0}, k_{N_{2}}^{0}, \ldots, k_{N_{r}}^{0}\right) \mid l_{s}=l_{s-1}=\cdots \\
& \left.=l_{2}=p^{n}-1, l_{1}=L_{1}\right\} .
\end{aligned}
$$

It is now easy to see that the total number of monomials $A$ in $\mathfrak{P}_{L_{1}}$ for which $A \notin A_{c}+J$ equals $\widetilde{D}_{1, M_{\max }^{0}}=\widetilde{S}_{M_{\max }^{0} 0}$ (the proof of this is similar to the proof of Lemma 23). Let

Fix an arbitrary integer $M_{1}$ such that $0 \leq M_{1}<Z_{1, \min }$.

$$
\begin{aligned}
\mathfrak{P}_{M_{1}}:=\{A & =N_{r}^{p^{n}-k_{N_{r}}^{0}} \cdots N_{1}^{p^{n}-k_{N_{1}}^{0}} Z_{s}^{l_{s}} \cdots Z_{1}^{l_{1}} P_{1}^{j_{1}} \cdots P_{t}^{j_{t}} \\
& \in \mathfrak{P}\left(k_{N_{1}}^{0}, k_{N_{2}}^{0}, \ldots, k_{N_{r}}^{0}\right) \mid l_{s}=l_{s-1}=\cdots \\
& \left.=l_{2}=p^{n}-1, l_{1}=M_{1}\right\} .
\end{aligned}
$$

Since $0 \leq M_{1}<Z_{1, \min }$, therefore any monomial $A \in \mathfrak{P}_{M_{1}}$ is divisible neither by [1] nor by [2]. Hence the total number of monomials $A$ in $\mathfrak{P}_{M_{1}}$ for which $A \notin A_{c}+J$ equals $p^{t n}$.

So if we define $\mathfrak{P}_{1}$ as the subset of $\mathfrak{P}\left(k_{N_{1}}^{0}, k_{N_{2}}^{0}, \ldots, k_{N_{r}}^{0}\right)$ consisting of all those monomials in $\mathfrak{P}\left(k_{N_{1}}^{0}, k_{N_{2}}^{0}, \ldots, k_{N_{r}}^{0}\right)$ for which $l_{s}=l_{s-1}=\cdots=l_{2}=p^{n}-1$, then the total number of monomials $A$ in the set $\mathfrak{P}_{1}$ for which $A \notin A_{c}+J$ 
equals $\mathfrak{S}_{M_{\max }^{0},}$. Similarly, if we define $\mathfrak{P}_{2}$ as the subset of $\mathfrak{P}\left(k_{N_{1}}^{0}, k_{N_{2}}^{0}, \ldots, k_{N_{r}}^{0}\right)$ consisting of all those monomials in it for which $l_{s}=l_{s-1}=\cdots=l_{3}=p^{n}-1$, then the total number of monomials $A$ in the set $\mathfrak{P}_{2}$ for which $A \notin A_{c}+J$ equals $\mathfrak{S}_{M_{\max }^{0}, 2}$. Proceeding inductively, it is now easy to see that the total number of monomials $A$ in the set $\mathfrak{P}\left(k_{N_{1}}^{0}, k_{N_{2}}^{0}, \ldots, k_{N_{r}}^{0}\right)$ for which $A \notin A_{c}+J$ equals $\mathfrak{S}_{M_{\max }^{0},}$,

Definition 25. Given any $M_{\max }$ in $M_{\max }[2]$, let $\Phi_{M_{\max }}:=$ $\mathbb{\mathfrak { S }}_{M_{\max }, s}$ where $\mathfrak{S}_{M_{\max }, s}$ is defined in the same way as $\mathfrak{S}_{M_{\max }^{0}, s}$ is defined in Lemma 24 (just replace $M_{\max }^{0}$ in Lemma 24 by $M_{\max }$ ).

4.2.5. The Formula for the Hilbert-Kunz Function. We will now state the formula for the Hilbert-Kunz function for the general binomial hypersurface that we have considered above. But for stating the formula, we need some more notation, which we provide first.

Set $G_{P_{(t-1)}}:=P_{t, \max }$ and for every $q \in\{1, \ldots,(t-2)\}$, let $G_{P_{q}}:=\left(p^{n}-P_{q+1, \max }\right) G_{P_{q+1}}+\left(P_{q+1, \max }\right) p^{n(t-q-1)}$;

Set $G_{Z_{1}}:=\left(p^{n}-P_{1, \max }\right) G_{P_{1}}+\left(P_{1, \max }\right) p^{n(t-1)}$, and for every $l \in\{2, \ldots, s\}$, let $G_{Z_{l}}:=\left(p^{n}-Z_{l-1, \max }\right) G_{Z_{l-1}}+$ $\left(Z_{l-1, \max }\right) p^{n(t+l-2)}$;

Set $G_{N_{1}}:=\left(p^{n}-Z_{s, \max }\right) G_{Z_{s}}+\left(Z_{s, \max }\right) p^{n(t+s-1)}$, and for every $i \in\{2, \ldots, r\}$, let $G_{N_{i}}:=\left(p^{n}-N_{i-1, \min }\right) G_{N_{i-1}}+$ $\left(N_{i-1, \min }\right) p^{n(t+s+i-2)}$.

Recall that for any $i \in\{1, \ldots, r\}$, let $a_{i}:=-\left(\Delta_{N_{i}}\right)$ and for any $i \in\{1, \ldots, r\}$ and any positive integer $n, M_{a_{i}, n}$ and $r_{a_{i}, n}$ are defined by the equation $p^{n}-1=a_{i} M_{a_{i}, n}+r_{a_{i}, n}$. Also recall the definitions of $E_{a_{i} b_{i}}$ and $p_{a_{i}}$ from Section 4.2.3.

For every $i \in\{1, \ldots, r\}$, let

$$
\begin{gathered}
D_{a_{i}, a_{r}}:=\left(1+E_{a_{i}, b_{i}}\right)+a_{i}\left(M_{a_{i}, n}-p_{a_{i}}-M_{a_{r}, n}+p_{a_{r}}\right) \\
a_{i}^{\prime}:=p^{n}-N_{i, \max }-\left[1+E_{a_{i}, b_{i}}+a_{i}\left(\widetilde{k}_{N_{i} \text { last }}-1\right)\right], \\
\widetilde{X}_{N_{i} \text { last }}:=M_{a_{r}, n}-p_{a_{r}}-M_{a_{i}, n}+p_{a_{i}}+\widetilde{k}_{N_{i} \text { last }} .
\end{gathered}
$$

Clearly $a_{i}^{\prime} \leq a_{i}$ for every $i \in\{1, \ldots, r\}$.

For any nonnegative integer $j$ such that $M_{a_{r}, n}-p_{a_{r}}-j$ belongs to the set $M_{\max }$ [2], let

$$
\int_{j}^{\left(N_{1}\right)}:=\Phi_{M_{a_{r}, n}-p_{a_{r}}-j}, \quad \text { for any } i \in\{2, \ldots, r\}
$$

and let

$$
\int_{j}^{\left(N_{i}\right)}:=\left\{\begin{aligned}
( & \left.D_{a_{i-1}, a_{r}}+j a_{i-1}\right) \int_{j}^{\left(N_{i-1}\right)}+a_{i-1} \int_{j+1}^{\left(N_{i-1}\right)}+\cdots \\
& +a_{i-1} \int_{\left(\widetilde{X}_{N_{i-1}, \text { last }}\right)-1}^{\left(N_{i-1}\right)}+a_{i-1}^{\prime} \int_{\widetilde{X}_{N_{i-1} \text { last }}}^{\left(N_{i-1}\right)} \\
& +\left(N_{i-1, \max }-N_{i-1, \min }\right) G_{N_{i-1}} \\
& +\left(N_{i-1, \min }\right) p^{n(s+t+i-2)} \quad \text { if } j<\widetilde{X}_{N_{i-1}, \text { last }} \\
\left(p^{n}-N_{i-1, \max }\right) \int_{j}^{\left(N_{i-1}\right)} & \\
& +\left(N_{i-1, \max }-N_{i-1, \min }\right) G_{N_{i-1}} \\
& +\left(N_{i-1, \min }\right) p^{n(s+t+i-2)} \quad \text { otherwise. }
\end{aligned}\right.
$$

Theorem 26. The formula for the Hilbert-Kunz function of the general binomial hypersurface under consideration is

$$
\begin{gathered}
D_{a_{r}, a_{r}} \int_{0}^{\left(N_{r}\right)}+a_{r} \int_{1}^{\left(N_{r}\right)}+\cdots+a_{r} \int_{\left(\widetilde{X}_{N_{r}, l a s t}\right)-1}^{\left(N_{r}\right)}+a_{r}^{\prime} \int_{\widetilde{X}_{N_{r}, \text { last }}}^{\left(N_{r}\right)} \\
\quad+\left(N_{r, \max }-N_{r, \min }\right) G_{N_{r}}+\left(N_{r, \min }\right) p^{n(s+t+r-1)} .
\end{gathered}
$$

Proof. Observe that for any fixed $i \in\{2, \ldots, r\}$ and any $j \in$ $\mathbb{Z}$ such that $0 \leq j \leq \widetilde{k}_{N_{r} \text {,last }}$, the number $\int_{j}^{\left(N_{i}\right)}$ provides an account of the number of monomials $A$ in $\mathfrak{M}$ which satisfy the following 3 properties simultaneously.

(i) $A \notin A_{c}+J$.

(ii) The power of each of the variables $N_{i+1}, \ldots, N_{r}$ is $p^{n}-$ 1.

(iii) The power of the variable $N_{i}$ is $p^{n}-k_{N_{i}}$ where

$$
\begin{gathered}
1+E_{a_{i}, b_{i}}+a_{i}\left(\widetilde{k}_{N_{i}}-1\right)<k_{N_{i}} \leq 1+E_{a_{i} b_{i}}+a_{i} \widetilde{k}_{N_{i}}, \\
\text { and } \widetilde{k}_{N_{i}}=M_{a_{i}, n}-p_{a_{i}}-M_{a_{r}, n}+p_{a_{r}}+j .
\end{gathered}
$$

Therefore for any $j \in \mathbb{Z}$ such that $0 \leq j \leq \widetilde{X}_{N_{r} \text {,last }}, \int_{j}^{\left(N_{r}\right)}$ denotes the number of monomials $A$ in $\mathfrak{M}$ for which $A \notin$ $A_{c}+J$ and the power of the variable $N_{r}$ is $p^{n}-k_{N_{r}}$ where $1+E_{a_{r}, b_{r}}+a_{r}(j-1)<k_{N_{r}} \leq 1+E_{a_{r}, b_{r}}+a_{r} j$.

Clearly then the number of monomials $A$ in $\mathfrak{M}$ for which $A \notin A_{c}+J$ and the power of the variable $N_{r}$ is $p^{n}-k_{N_{r}}$ where $1 \leq k_{N_{r}} \leq 1+E_{a_{r}, b_{r}}$ equals $D_{a_{r}, a_{r}} \int_{0}^{\left(N_{r}\right)}$. Similarly, the number of monomials $A$ in $\mathfrak{M}$ for which $A \notin A_{c}+J$ and the power of the variable $N_{r}$ is $p^{n}-k_{N_{r}}$ where $1+E_{a_{r}, b_{r}}+a_{r}(j-1)<$ $k_{N_{r}} \leq 1+E_{a_{r}, b_{r}}+a_{r} j$ and $1 \leq j \leq\left(\widetilde{X}_{N_{r}, \text { last }}\right)-1$ equals $a_{r} \int_{j}^{\left(N_{r}\right)}$. We can similarly account for the terms $a_{r}^{\prime} \int_{\widetilde{X}_{N_{r} \text { last }}}^{\left(N_{r}\right)},\left(N_{r, \text { max }}-\right.$ $\left.N_{r, \text { min }}\right) G_{N_{r}}$, and $\left(N_{r, \text { min }}\right) p^{n(s+t+r-1)}$ in the formula (45) of the Hilbert-Kunz function.

4.3. An Illustrative Example. For this example as well as for the example given in Section 4.4 below, we will need the following notation. 
Given any real number $\beta$, let $\langle\beta\rangle$ denote the smallest integer $\geq \beta$ and let $[\beta]$ denote the largest integer $\leq \beta$. We call $\langle\beta\rangle$ and $[\beta]$ the upper and the lower integral parts of $\beta$.

Let $S=k\left[N_{2}, N_{1}, Z_{2}, Z_{1}, P_{1}, P_{2}\right]$ where $k$ is a field of arbitrary prime characteristic $p>0$, and let $J$ be the ideal in $S$ defined by $J=(f)$ where $f=N_{2}^{10} N_{1}^{6} Z_{2}^{5} Z_{1}^{2} P_{1}^{3} P_{2}^{4}+$ $N_{2}^{4} N_{1}^{2} Z_{2}^{5} Z_{1}^{2} P_{1}^{6} P_{2}^{9}$. The differences $\Delta_{N_{2}}, \Delta_{N_{1}}, \Delta_{Z_{2}}, \Delta_{Z_{1}}, \Delta_{P_{1}}$, and $\Delta_{P_{2}}$ are therefore given by $-6,-4,0,0,3,5$, respectively. Hence the $N_{i}$ 's are the negative difference variables, the $Z_{i}$ 's are the zero difference variables, and the $P_{i}$ 's are the positive difference variables. Here $a_{1}=-\Delta_{N_{1}}=4, a_{2}=-\Delta_{N_{2}}=6$, $b_{1}=N_{1, \min }=2$, and $b_{2}=N_{2, \min }=4$. Let $E_{a_{1}, b_{1}}, E_{a_{2}, b_{2}}$ be as defined in Section 4.2.3 above. Here, $E_{a_{1}, b_{1}}=E_{4,2}$ and $E_{a_{2}, b_{2}}=E_{6,4}$. For any $i \in\{1,2\}$, let $M_{a_{i}, n}$ and $r_{a_{i}, n}$ be defined by the equation(s) $p^{n}-1=a_{i} M_{a_{i}, n}+r_{a_{i}, n}$. That is,

$$
\begin{gathered}
M_{a_{1}, n}=M_{4, n}=\frac{p^{n}-1-r_{4, n}}{4}, \\
M_{a_{2}, n}=M_{6, n}=\frac{p^{n}-1-r_{6, n}}{6}, \\
\text { Also } \widetilde{k}_{N_{1}, \text { last }}=\left\langle\frac{p^{n}-6-1-E_{a_{1}, b_{1}}}{4}\right\rangle, \\
\widetilde{k}_{N_{2}, \text { last }}=\left\langle\frac{p^{n}-10-1-E_{a_{2}, b_{2}}}{6}\right\rangle,
\end{gathered}
$$

the set $M_{\max }[2]=\left\{M_{6, n}-p_{6}-j \mid j \in \mathbb{Z}, 0 \leq j \leq \widetilde{k}_{N_{2}, \text { last }}\right\}$.

For every $j \in \mathbb{Z}$ such that $0 \leq j \leq \widetilde{k}_{N_{2} \text {,last }}$, we have

$$
\begin{aligned}
\operatorname{Min}_{P_{1}, M_{6, n}-p_{6}-j}^{c} & :=\operatorname{Min}\left\{p^{n}-3,\left(M_{6, n}-p_{6}-j\right) \Delta_{P_{1}}\right\} \\
& =3\left(M_{6, n}-p_{6}-j\right), \\
\operatorname{Min}_{P_{2}, M_{6, n}-p_{6}-j}^{c} & :=\operatorname{Min}\left\{p^{n}-4,\left(M_{6, n}-p_{6}-j\right) \Delta_{P_{2}}\right\} \\
& =5\left(M_{6, n}-p_{6}-j\right) .
\end{aligned}
$$

Using the formulae given in Lemma 24, it follows that

$$
\begin{aligned}
\mathfrak{S}_{M_{6, n}-p_{6}-j, 0}= & 15 p^{n}-30\left(M_{6, n}-p_{6}-j\right)-54, \\
\mathfrak{S}_{M_{6, n}-p_{6}-j, 1}= & {\left[15 p^{n}-30\left(M_{6, n}-p_{6}-j\right)-54\right] } \\
& \times\left(p^{n}-2\right)+2 p^{2 n}, \\
\mathfrak{S}_{M_{6, n}-p_{6}-j, 2}= & {\left[15 p^{n}-30\left(M_{6, n}-p_{6}-j\right)-54\right] } \\
& \times\left(p^{n}-2\right)\left(p^{n}-5\right)+7 p^{3 n}-10 p^{2 n} .
\end{aligned}
$$

Since for any $j \in \mathbb{Z}$ such that $0 \leq j \leq \widetilde{k}_{N_{2} \text {,last }}$, we know that $\int_{j}^{\left(N_{1}\right)}:=\mathfrak{S}_{M_{6, n}-p_{6}-j, s}$ and $s=$ the total number of zero difference variables $=2$ here, therefore we have

$$
\begin{aligned}
\int_{j}^{\left(N_{1}\right)}= & {\left[15 p^{n}-30\left(M_{6, n}-p_{6}-j\right)-54\right] } \\
& \times\left(p^{n}-2\right)\left(p^{n}-5\right)+7 p^{3 n}-10 p^{2 n} .
\end{aligned}
$$

For getting the formula for the Hilbert-Kunz function in this case, we need to compute $\int_{j}^{\left(N_{2}\right)}$ for the various values of $j$ under consideration. A straight forward computation tells us that for any $j \in \mathbb{Z}$ such that $0 \leq j<\widetilde{X}_{N_{1} \text {, last }}$, we have

$$
\begin{aligned}
\int_{j}^{\left(N_{2}\right)}= & \left(D_{a_{1}, a_{2}}+4 j\right) \int_{j}^{\left(N_{1}\right)}+4 \sum_{k=j+1}^{\widetilde{X}_{N_{1} \text { last }}-1} \int_{k}^{\left(N_{1}\right)} \\
& +a_{1}^{\prime} \int_{\widetilde{X}_{N_{1}, \text { last }}}^{\left(N_{1}\right)}+(6-2) G_{N_{1}}+2 p^{n(2+2+2-2)},
\end{aligned}
$$

where

$$
\begin{gathered}
D_{a_{1}, a_{2}}=\left(1+E_{a_{1}, b_{1}}\right)+4\left(M_{4, n}-p_{4}-M_{6, n}+p_{6}\right) \\
=\left(1+E_{4,2}\right)+4\left(M_{4, n}-p_{4}-M_{6, n}+p_{6}\right) \\
a_{1}^{\prime}=p^{n}-6-1-E_{a_{1}, b_{1}}-4\left(\widetilde{k}_{N_{1} \text { last }}-1\right), \\
\widetilde{X}_{N_{1}, \text { last }}=M_{6, n}-p_{6}-M_{4, n}+p_{4}+\widetilde{k}_{N_{1}, \text { last }}, \\
G_{N_{1}}=19 p^{3 n}-130 p^{2 n}+372 p^{n}-360 .
\end{gathered}
$$

Similarly, for any $j \in \mathbb{Z}$ such that $j \geq \widetilde{X}_{N_{1} \text {,last }}$, we have

$$
\begin{aligned}
\int_{j}^{\left(N_{2}\right)}= & \left(p^{n}-6\right) \int_{j}^{\left(N_{1}\right)}+(6-2) G_{N_{1}}+2 p^{n(2+2+2-2)} \\
=\left(p^{n}-6\right) & {\left[\left(15 p^{n}-30\left(M_{6, n}-p_{6}-j\right)-54\right)\right.} \\
& \left.\times\left(p^{n}-2\right)\left(p^{n}-5\right)+7 p^{3 n}-10 p^{2 n}\right] \\
& +4\left[19 p^{3 n}-130 p^{2 n}+372 p^{n}-360\right]+2 p^{4 n} .
\end{aligned}
$$

Clearly then $\int_{j}^{\left(N_{2}\right)}$ is a rational function in $p$ and $n$ for all values of $j$. Therefore, the formula for the corresponding Hilbert-Kunz function is

$$
\begin{aligned}
& D_{a_{2}, a_{2}} \int_{0}^{\left(N_{2}\right)}+6 \sum_{j=1}^{\widetilde{X}_{N_{2}, \text { last }}-1} \int_{j}^{\left(N_{2}\right)}+a_{2}^{\prime} \int_{\widetilde{X}_{N_{2}, \text { last }}}^{\left(N_{2}\right)} \\
& +(10-4) G_{N_{2}}+4 p^{n(2+2+2-1)},
\end{aligned}
$$

where

$$
\begin{gathered}
D_{a_{2}, a_{2}}=1+E_{a_{2}, b_{2}}=1+E_{6,4}, \\
a_{2}^{\prime}=p^{n}-10-1-E_{a_{2}, b_{2}}-6\left(\widetilde{k}_{N_{2}, \text { last }}-1\right), \\
\widetilde{X}_{N_{2}, \text { last }}=M_{6, n}-p_{6}-M_{4, n}+p_{4}+\widetilde{k}_{N_{2}, \text { last}}, \\
G_{N_{2}}=21 p^{4 n}-168 p^{3 n}+632 p^{2 n}-1104 p^{n}+720, \\
\text { the formulae of } \int_{j}^{\left(N_{2}\right)} \text { are given above. }
\end{gathered}
$$

Since all the quantities involved in the above formula for the Hilbert-Kunz function are rational functions in the variables $p$ and $n$, therefore the Hilbert-Kunz function is a rational function in $p$ and $n$. 
4.4. Example of the One-Dimensional Case. In this subsection, we will discuss the case of 1-dimensional binomial hypersurfaces and will observe that using the above formula for the Hilbert-Kunz function, we do get that in this case of 1-dimension, the associated Hilbert-Kunz multiplicity is an integer (e.g., see Chapter 6, Corollary 6.2 of [7]). In this case, this integer happens to be equal to the ordinary multiplicity because the Hilbert-Kunz multiplicity is equal to the ordinary multiplicity for 1-dimensional rings.

In the case of 1-dimensional binomial hypersurfaces, we have $S=k\left[x_{1}, x_{2}\right], J=(f)$ where $f=[2]+[1], R=S / J$ and the terms [2] and [1] of $f$ are monomials in the 2 variables $x_{1}$ and $x_{2}$.

There are 3 possible cases:

(1) $x_{1} \triangleright x_{2}$ where $x_{1}$ is a negative difference variable and $x_{2}$ is a positive difference variable;

(2) $x_{1} \triangleright x_{2}$ where $x_{1}$ is a negative difference variable and $x_{2}$ is a zero difference variable;

(3) $x_{1} \triangleright x_{2}$ where $x_{1}$ is a zero difference variable and $x_{2}$ is a positive difference variable.

We will first discuss Cases (2) and (3).

In Cases (2) and (3), it is easy to check (without using the formula above) following.

The Hilbert-Kunz function evaluated at $p^{n}=\left(x_{1, \min }+\right.$ $\left.x_{2, \min }\right) p^{n}-x_{1, \min } x_{2, \min }$ and The associated HilbertKunz multiplicity $=x_{1, \min }+x_{2, \min }$ which is an integer.

We will now discuss Case (1).

Since $x_{1}$ is a negative difference variable and $x_{2}$ is a positive difference variable, then using our notation in Section 4.2.1, we can denote $x_{1}$ by $N_{1}$ and $x_{2}$ by $P_{1}$. Then writing $x_{1} \triangleright x_{2}$ is the same as writing $N_{1} \triangleright P_{1}$. In this case, there are 3 subcases.

Subcase 1.1 (when $a_{1}<\Delta_{P_{1}}$ ). It is an exercise to check that using the above formula for the Hilbert-Kunz function, we get that for $n$ large enough.

The Hilbert-Kunz function evaluated at $p^{n}$ equals

$$
\begin{aligned}
& \left(1+E_{a_{1}, b_{1}}\right) P_{1, \min }+a_{1} P_{1, \min } \\
& \times\left[p^{n} \frac{\left(\Delta_{P_{1}}-a_{1}\right)}{a_{1} \Delta_{P_{1}}}-\frac{1}{a_{1}}-\frac{r_{a_{1}, n}}{a_{1}}-p_{a_{1}}+\frac{P_{1, \min }}{\Delta_{P_{1}}}\right] \\
& -a_{1} P_{1, \max }+a_{1} P_{1, \max }\left\langle\frac{p^{n}-N_{1, \max }-1-E_{a_{1}, b_{1}}}{a_{1}}\right\rangle \\
& -a_{1} P_{1, \max }\left[p^{n} \frac{\left(\Delta_{P_{1}}-a_{1}\right)}{a_{1} \Delta_{P_{1}}}-\frac{1}{a_{1}}-\frac{r_{a_{1}, n}}{a_{1}}-p_{a_{1}}+\frac{P_{1, \min }}{\Delta_{P_{1}}}\right] \\
& +a_{1}^{\prime} P_{1, \max }+\left(N_{1, \max }-N_{1, \min }\right) P_{1, \max }+\left(N_{1, \min }\right) p^{n},
\end{aligned}
$$

where for any real number $\beta,\langle\beta\rangle$ and $[\beta]$ denote the upper and lower integral parts of $\beta$, respectively.
Hence the associated Hilbert-Kunz multiplicity equals

$$
a_{1} P_{1, \min } \frac{\left(\Delta_{P_{1}}-a_{1}\right)}{a_{1} \Delta_{P_{1}}}+\frac{a_{1} P_{1, \max }}{\Delta_{P_{1}}}+N_{1, \text { min }}
$$

which is nothing but $P_{1, \min }+a_{1}+N_{1, \min }$, which is an integer.

Subcase 1.2 (when $a_{1}>\Delta_{P_{1}}$ ). It is an exercise to check that using the above formula for the Hilbert-Kunz function, we get that for $n$ large enough

the Hilbert-Kunz function evaluated at $p^{n}$ equals

$\left(p^{n}-N_{1, \text { max }}\right) P_{1, \text { max }}+\left(N_{1, \text { max }}-N_{1, \text { min }}\right) P_{1, \text { max }}+\left(N_{1, \text { min }}\right) p^{n}$.

Hence the associated Hilbert-Kunz multiplicity equals $P_{1, \max }+N_{1, \text { min }}$, which is an integer.

Subcase 1.3 (when $a_{1}=\Delta_{P_{1}}$ ). Let $\widetilde{N}_{0}$ be the smallest positive integer such that if $\widetilde{k}>\widetilde{N}_{0}$, then $P_{1, \min }-1-r_{a_{1}, n}-a_{1} p_{a_{1}}<a_{1} \widetilde{k}$. Similarly, let $\widetilde{N}_{0}^{\prime}$ be the smallest positive integer such that if $\widetilde{k}>\widetilde{N}_{0}^{\prime}$, then $P_{1, \max }-1-r_{a_{1}, n}-a_{1} p_{a_{1}}<a_{1} \widetilde{k}$. Clearly then $\widetilde{N}_{0}^{\prime} \geq \widetilde{N}_{0}$.

It is an exercise to check that using the above formula for the Hilbert-Kunz function, we get that for $n$ large enough, the Hilbert-Kunz function evaluated at $p^{n}$ equals

$$
\begin{aligned}
& \left(1+E_{a_{1}, b_{1}}\right) P_{1, \text { min }}+a_{1} P_{1, \min } \widetilde{N}_{0} \\
& +a_{1} \sum_{\widetilde{k}=\widetilde{N}_{0}+1}^{\widetilde{N}_{0}^{\prime}}\left(1+r_{a_{1}, n}+a_{1} p_{a_{1}}+a_{1} \tilde{k}\right) \\
& +a_{1} P_{1, \max }\left(\left\langle\frac{p^{n}-N_{1, \max }-1-E_{a_{1}, b_{1}}}{a_{1}}\right\rangle-1-\widetilde{N}_{0}^{\prime}\right) \\
& +a_{1}^{\prime} P_{1, \max }+\left(N_{1, \text { max }}-N_{1, \text { min }}\right) P_{1, \text { max }}+\left(N_{1, \text { min }}\right) p^{n},
\end{aligned}
$$

where for any real number $\beta,\langle\beta\rangle$ denotes the upper integral part of $\beta$.

Hence the associated Hilbert-Kunz multiplicity equals $P_{1, \text { max }}+N_{1, \text { min }}$, which is an integer.

4.5. Rationality of the Hilbert-Kunz Multiplicity. The HilbertKunz multiplicity for the general Binomial hypersurface (whose Hilbert-Kunz function is given by (45) above) is by definition equal to the coefficient of $p^{n(s+t+r-1)}$ in the formula given by (45). We can easily see from the above discussion that for any $j$ such that $0 \leq j \leq \widetilde{X}_{N_{r}}$,ast, $\int_{j}^{\left(N_{r}\right)}$ is obtained iteratively by first computing $\int_{j}^{\left(N_{1}\right)}$ for all $j$, then $\int_{j}^{\left(N_{2}\right)}$ (for all $\left.j\right), \ldots$, and so on till $\int_{j}^{\left(N_{r-1}\right)}$ for all $j$. Moreover, for any nonnegative integer $j$ such that $0 \leq j \leq \widetilde{k}_{N_{r}, \text { last }}$, we know that $M_{a_{r}, n}-p_{a_{r}}-j$ belongs to the set $M_{\max }[2]$ and

$$
\int_{j}^{\left(N_{1}\right)}:=\Phi_{M_{a_{r}, n}-p_{a_{r}}-j}=\widetilde{S}_{M_{a_{r}, n}-p_{a_{r}}-j, s} .
$$


So let us first give a formula for $\mathfrak{S}_{M_{a_{r}, n}-p_{a_{r}}-j, s}$. I will rather give a formula for $\widetilde{S}_{M_{\max }, s}$ for any $M_{\max }$ in $M_{\max }$ [2] in general and then one can put $M_{\max }=M_{a_{r}, n}-p_{a_{r}}-j$ in it to get the required formula for $\mathfrak{S}_{M_{a_{r}, n}-p_{a_{r}-j, s}}$.

Let $M_{\max } \in M_{\max }$ [2] be arbitrary. To compute $\mathfrak{S}_{M_{\max }, s}$, we first need to compute $\mathfrak{S}_{M_{\max }, 0}$. I will compute $\mathfrak{S}_{M_{\max }, 0}$ only in the most general case, that is, when

$$
\begin{gathered}
\operatorname{Min}_{P_{q}, M_{\text {max }}}^{c}<p^{n}-P_{q, \text { max }}<p^{n}-P_{q, \text { min }} \\
\forall q \in\{1, \ldots, t\}, \forall M_{\text {max }} \in M_{\text {max }}[2] .
\end{gathered}
$$

In this case, we have

$$
\operatorname{Min}_{P_{q}, M_{\max }}^{c}=M_{\max } \Delta_{P_{q}} .
$$

In all other cases, it is a tedious job to compute $\mathfrak{S}_{M_{\max }, 0}$ because we will have to take care of various complicated possibilities like

for some $q \in\{1, \ldots, t\}$, we may have $\operatorname{Min}_{P_{q}, M_{\max }}^{c}<$ $p^{n}-P_{q, \min }$ and for some other $q^{\prime} \in\{1, \ldots, t\}$, we may have $\operatorname{Min}_{P_{q^{\prime}}, M_{\max }}^{c}=p^{n}-P_{q^{\prime}, \min }$.

This makes the computation of $\mathfrak{S}_{M_{\operatorname{mx}, 0}}$ highly tedious and complicated. Let us now compute ${\stackrel{\mathscr{S}}{M_{\text {max }}, 0}}_{\text {for }}$ for most general case.

For $2 \leq l \leq t$, let $E_{l, \min }$ denote the sum of all possible terms which are products of $l$-many items either of the type $M_{\max } \Delta_{P_{q}}$ or of the type $P_{q, \min }$ where $q \in\{1, \ldots, t\}$ such that the following two properties hold.

(i) At least one of the $l$ items in each such product is of the type $M_{\max } \Delta_{P_{q}}$;

(ii) not all of the $l$ items in any such product are of the type $M_{\max } \Delta_{P_{q}}$.

Similarly, for any $l \in\{2, \ldots, t\}$, let $E_{l, \max }$ denote the sum of all possible terms which are products of $l$-many items either of the type $M_{\max } \Delta_{P_{q}}$ or of the type $P_{q, \max }$ where $q \in\{1, \ldots, t\}$ such that the following two properties hold.

(i) At least one of the $l$ items in each such product is of the type $M_{\max } \Delta_{P_{q}}$;

(ii) not all of the $l$ items in any such product are of the type $M_{\max } \Delta_{P_{q}}$.

For $2 \leq l \leq t$, let $T_{l}$ denote the sum of all possible terms which are products of the type $\prod_{\substack{i_{1}, i_{2}, \ldots, i_{i} \in\{1, \ldots, t\} \\ i_{1} \neq i_{2} \neq \cdots \neq i_{l}}} P_{i_{1}, \max } P_{i_{2} \text {, max }} \cdots P_{i_{l} \text {, max }}$.

Then

$$
\begin{aligned}
\mathfrak{S}_{M_{\max }, 0}= & p^{(t-1) n}\left[\sum_{i=1}^{t} P_{i, \max }\right] \\
& +\sum_{l=2}^{t}(-1)^{l} p^{(t-l) n}\left\{E_{l, \min }-E_{l, \max }-T_{l}\right\} .
\end{aligned}
$$

Hence the formula for $\Phi_{M_{\max }}\left(:=\mathfrak{S}_{M_{\max }, s}\right)$ is given by

$$
\begin{aligned}
& {\left[p^{(t-1) n}\left[\sum_{i=1}^{t} P_{i, \max }\right]+\sum_{l=2}^{t}(-1)^{l} p^{(t-l) n}\right.} \\
& \left.\times\left\{E_{l, \min }-E_{l, \max }-T_{l}\right\}\right]\left[\prod_{i=1}^{s}\left(p^{n}-Z_{i, \min }\right)\right] \\
& +\sum_{k=1}^{s}\left[(-1)^{k+1} p^{(t+s-k) n}\right. \\
& \left.\times\left(\sum_{\substack{i_{1}, i_{i}, \ldots, i_{k} \in\{1, \ldots, s\} \\
i_{1} \neq i_{2} \neq \cdots \neq i_{k}}} Z_{i_{1}, \min } Z_{i_{2}, \min } \cdots Z_{i_{k}, \min }\right)\right] .
\end{aligned}
$$

Since $\int_{j}^{\left(N_{1}\right)}=\Phi_{M_{a_{r}, n}-p_{a_{r}}-j}$, we get the formula for $\int_{j}^{\left(N_{1}\right)}$ from (64) above by putting $M_{\max }=M_{a_{r}, n}-p_{a_{r}}-j$ in it.

Let us now write down the formula (45) for the HilbertKunz function, in a different way which will help us see how the formula for the Hilbert-Kunz multiplicity will look like. But for that, we first need some notation.

For each $j \in \mathbb{N}$, let $F_{j}^{(1)}:=1, F_{j}^{(2)}:=2 j-1$ and for any positive integer $k \geq 3$, let $F_{j}^{(k)}:=\sum_{l=1}^{j-1} F_{l}^{(k-1)}+j F_{j}^{(k-1)}$.

For each $0 \leq j \leq \widetilde{X}_{N_{r} \text { last }}$, let $C_{j}^{\left(N_{r}\right)}$ denote the coefficient of $\int_{j}^{\left(N_{r}\right)}$ in the formula (45) for the HilbertKunz function.

For any $m \in\{1, \ldots, r\}$, let $V^{(m)}:=\left\{D_{a_{r}, a_{r}}, D_{a_{r-1}, a_{r}}, \ldots\right.$, $\left.D_{a_{m}, a_{r}}\right\}, U^{(m)}:=\left\{a_{r}, a_{r-1}, \ldots, a_{m}\right\}$, and $C_{0}^{\left(N_{m}\right)}:=$ $\prod_{k=m}^{r} D_{a_{k}, a_{r}}$.

For any integer $j$ such that $1 \leq j<\widetilde{X}_{N_{m} \text {, last }}$ and any integer $l$ such that $1 \leq l \leq r-m+1$, let $H_{l}^{(m)}:=$ sum of all possible terms which are products of $r-l-m+1$ many $D_{a_{k}, a_{r}}$ s and $l$ many $a_{k}$ s where the $D_{a_{k}, a_{r}}$ s belong to the set $V^{(m)}$ and the $a_{k}$ s belong to the set $U^{(m)}$.

Let $m \in\{1, \ldots, r-1\}$ be arbitrary. Then for any integer $j$ such that $1 \leq j<\widetilde{X}_{N_{m}, \text { last }}$, let

$$
C_{j}^{\left(N_{m}\right)}:=\sum_{l=1}^{r-m+1} F_{j}^{(l)} H_{l}^{(m)} .
$$

And for any integer $j$ such that $\widetilde{X}_{N_{m} \text {, last }} \leq j \leq \widetilde{X}_{N_{r} \text {, last }}$, let us define $C_{j}^{\left(N_{m}\right)}$ inductively as follows.

Let $C_{\widetilde{X}_{N_{m}, \text { last }}}^{\left(N_{m}\right)}:=\quad a_{m}^{\prime}\left[\Sigma_{j=0}^{\widetilde{X}_{N_{m} \text { last }}-1} C_{j}^{\left(N_{m+1}\right)}\right]+\left(p^{n}-\right.$ $\left.N_{m, \max }\right) C_{\widetilde{X}_{N_{m}, \text { last }}}^{\left(N_{N_{m}, \text { last }}\right.}$ and for any $\widetilde{X}_{N_{m} \text {, last }}<j \leq \widetilde{X}_{N_{r} \text {, last }}$, let $C_{j}^{\left(N_{m}\right)}:=\left(p^{n}-N_{m, \max }\right) C_{j}^{\left(N_{m+1}\right)}$. 
Now if we expand the formula ((45) above) of the HilbertKunz function, assuming that $\widetilde{X}_{N_{1}, \text { last }} \leq \widetilde{X}_{N_{2} \text {,last }} \leq \cdots \leq$ $\widetilde{X}_{N_{r} \text {, last }}$, we get that the expression in (45) equals

$$
\begin{aligned}
& \sum_{j=0}^{\left(\widetilde{X}_{N_{1}, \text { last }}\right)-1} C_{j}^{\left(N_{1}\right)} \int_{j}^{\left(N_{1}\right)} \\
& +\left[a_{1}^{\prime}\left(\sum_{j=0}^{\left(\widetilde{X}_{N_{1}, \text { last }}\right)-1} C_{j}^{\left(N_{2}\right)}\right)+\left(p^{n}-N_{1, \max }\right) C_{\widetilde{X}_{N_{1}, \text { last }}^{\left(N_{2}\right)}}^{\left(N^{2}\right.}\right] \int_{\widetilde{X}_{N_{1}, \text { last }}}^{\left(N_{1}\right)} \\
& +\left(p^{n}-N_{1, \max }\right) \sum_{j=\left(\widetilde{X}_{N_{1}, \text { last }}\right)+1}^{\widetilde{X}_{N_{r} \text {, last }}} C_{j}^{\left(N_{2}\right)} \int_{j}^{\left(N_{1}\right)} \\
& +\left(N_{r, \text { max }}-N_{r, \text { min }}\right) G_{N_{r}}+N_{r, \text { min }} p^{n(s+t+r-1)} \\
& +\sum_{k=1}^{r-1}\left[\left(N_{r-k, \max }-N_{r-k, \min }\right) G_{N_{r-k}}\right. \\
& \left.+N_{r-k, \min } p^{n(s+t+r-k-1)}\right] \\
& \times\left[C_{0}^{\left(N_{r-k+1}\right)}+\sum_{l=1}^{k}\left(H_{l}^{(r-k+1)} \sum_{j=1}^{\left(\widetilde{X}_{N_{r-k+1} \text { last }}\right)-1} F_{j}^{(l)}\right)\right. \\
& \left.+\sum_{j=\widetilde{X}_{N_{r-k+1}, \text { last }}}^{\widetilde{X}_{N_{r} \text { last }}} C_{j}^{\left(N_{r-k+1}\right)}\right]
\end{aligned}
$$

where the formulae for $\int_{j}^{\left(N_{1}\right)}$ can be obtained from (64). The Hilbert-Kunz multiplicity is by definition the coefficient of $p^{n(s+t+r-1)}$ in the expression (66) above. Computing the coefficient of $p^{n(s+t+r-1)}$ in the expression (66) above, we get that the Hilbert-Kunz multiplicity equals

$$
\begin{gathered}
N_{r, \text { min }}+\sum_{k=1}^{r-1} N_{r-k, \text { min }}\left[\text { Coefficient of } p^{k n}\right. \text { in } \\
\left.\sum_{l=1}^{k} H_{l}^{(r-k+1)}\left(\sum_{j=1}^{\widetilde{X}_{N_{r-k+1} \text {,ast }}-1} F_{j}^{(l)}\right)\right] \\
+\sum_{q=0}^{t-1}\left\{\left[\text { coefficient of } j^{q} p^{n(t+s-1-q)} \text { in } \int_{j}^{\left(N_{1}\right)}\right]\right. \\
\times\left\{\begin{array}{c}
{\left[\text { terms of } H_{1}^{(1)} \text { of degree } p^{n(r-1)}\right]} \\
\times \sum_{j=1}^{\widetilde{X}_{N_{1} \text { last }}-1} j^{q} F_{j}^{(1)}
\end{array}\right.
\end{gathered}
$$

$+\left[\right.$ terms of $H_{2}^{(1)}$ of degree $\left.p^{n(r-2)}\right]$

$\times \sum_{j=1}^{\widetilde{X}_{N_{1}, \text { last }}-1} j^{q} F_{j}^{(2)}+\cdots$

$+\left[\right.$ terms of $H_{r}^{(1)}$ of degree $\left.p^{n(r-r)}\right]$

$\left.\left.\times \sum_{j=1}^{\widetilde{X}_{N_{1} \text { last }}-1} j^{q} F_{j}^{(r)}\right\}\right\}$.

If we expand the above formula (expression (67)) of the Hilbert-Kunz multiplicity, then inside the expansion, we will get terms which are of the form:

(i) finite (by "finite", I mean depending upon $n$ ) sums of the form $\sum_{j=\epsilon_{n}}^{\theta_{n}} j^{v}$ where $v$ is some positive integer and $\epsilon_{n}$ and $\theta_{n}$ are upper or lower integral parts of some real numbers which are of the form "polynomials in $p^{n}$ with rational coefficients";

(ii) polynomials in $p^{n}$ with rational coefficients (including constant terms which are rational numbers);

(iii) products of the above mentioned 2 types of terms.

It hence follows from the above discussion that the Hilbert-Kunz multiplicity associated with any general binomial hypersurface is always rational.

\section{Conflict of Interests}

The author declares that there is no conflict of interests regarding the publication of this paper.

\section{References}

[1] P. Monsky, "The Hilbert-Kunz function," Mathematische Annalen, vol. 263, no. 1, pp. 43-49, 1983.

[2] A. Conca, "Hilbert-Kunz function of monomial ideals and binomial hypersurfaces," Manuscripta Mathematica, vol. 90, no. 3, pp. 287-300, 1996.

[3] R. O. Buchweitz and Q. Chen, "Hilbert-Kunz functions of cubic curves and surfaces," Journal of Algebra, vol. 197, no. 1, pp. 246267, 1997.

[4] N. Fakhruddin and V. Trivedi, "Hilbert-Kunz functions and multiplicities for full flag varieties and elliptic curves," Journal of Pure and Applied Algebra, vol. 181, no. 1, pp. 23-52, 2003.

[5] K. Eto, "Multiplicity and Hilbert-Kunz multiplicity of monoid rings," Tokyo Journal of Mathematics, vol. 25, no. 2, pp. 241-245, 2002.

[6] K. Watanabe, "Hilbert-Kunz multiplicity of toric rings," Proceedings of the Institute of Natural Sciences, Nihon UNiversity, no. 35,2000 .

[7] C. Huneke, Tight Closure and Its Applications, CBMS Regional Conference Series in Mathematics, No. 88, American Mathematical Society, 1996. 


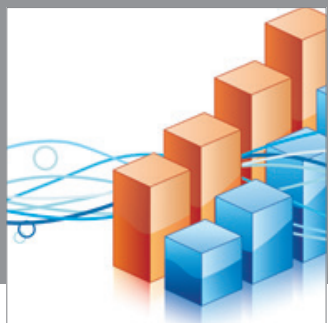

Advances in

Operations Research

mansans

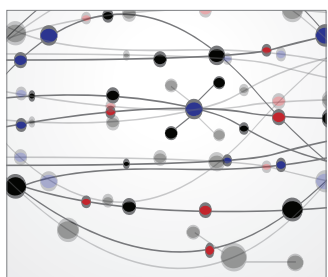

The Scientific World Journal
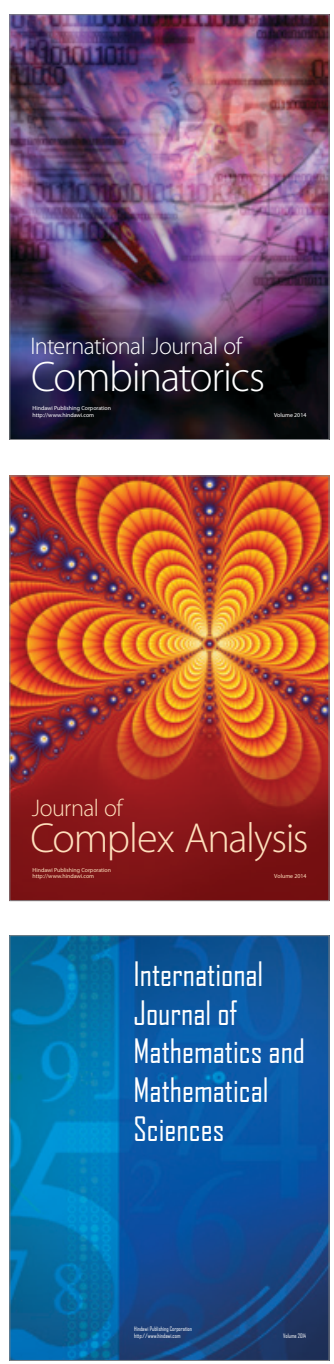
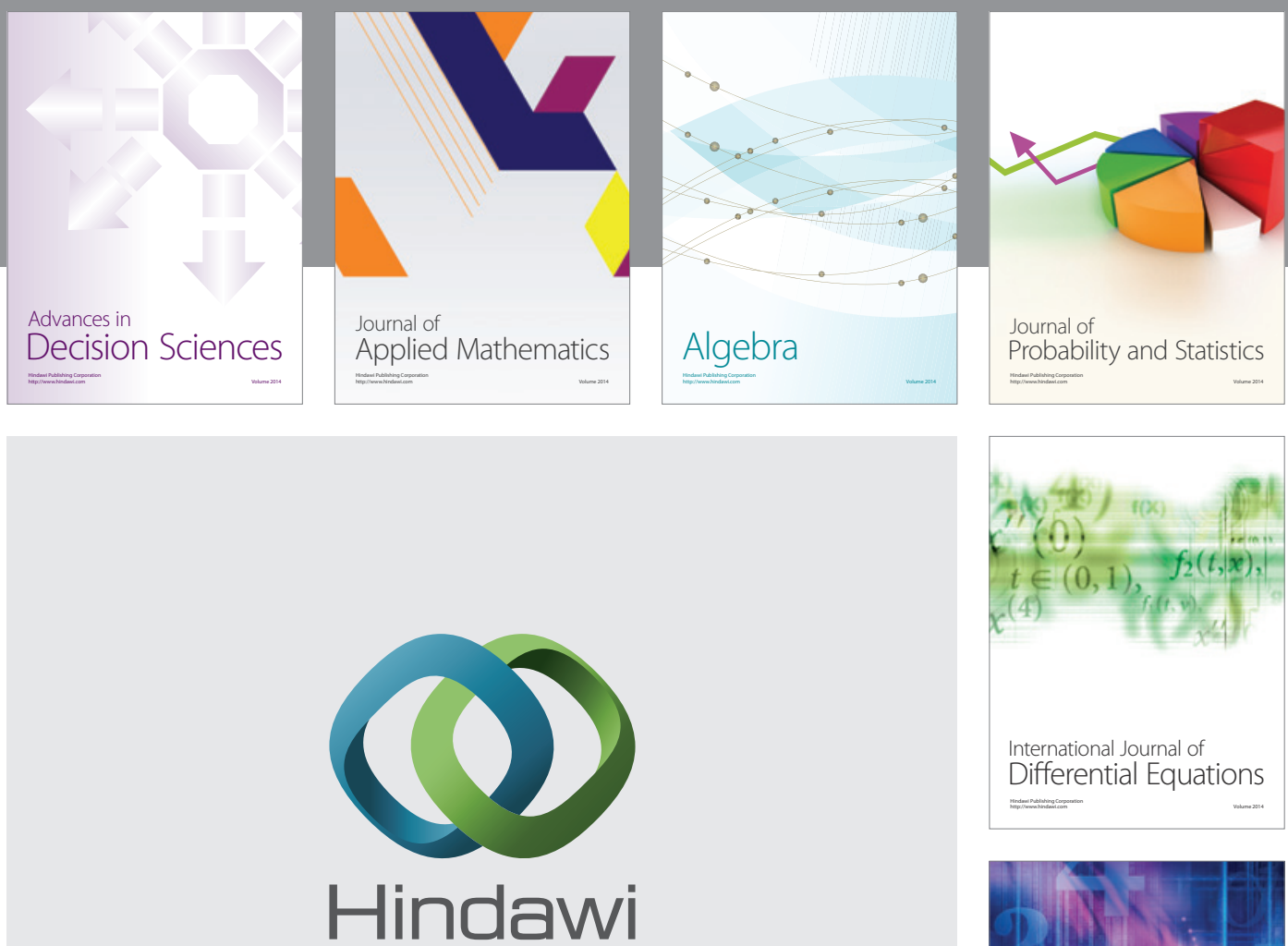

Submit your manuscripts at http://www.hindawi.com
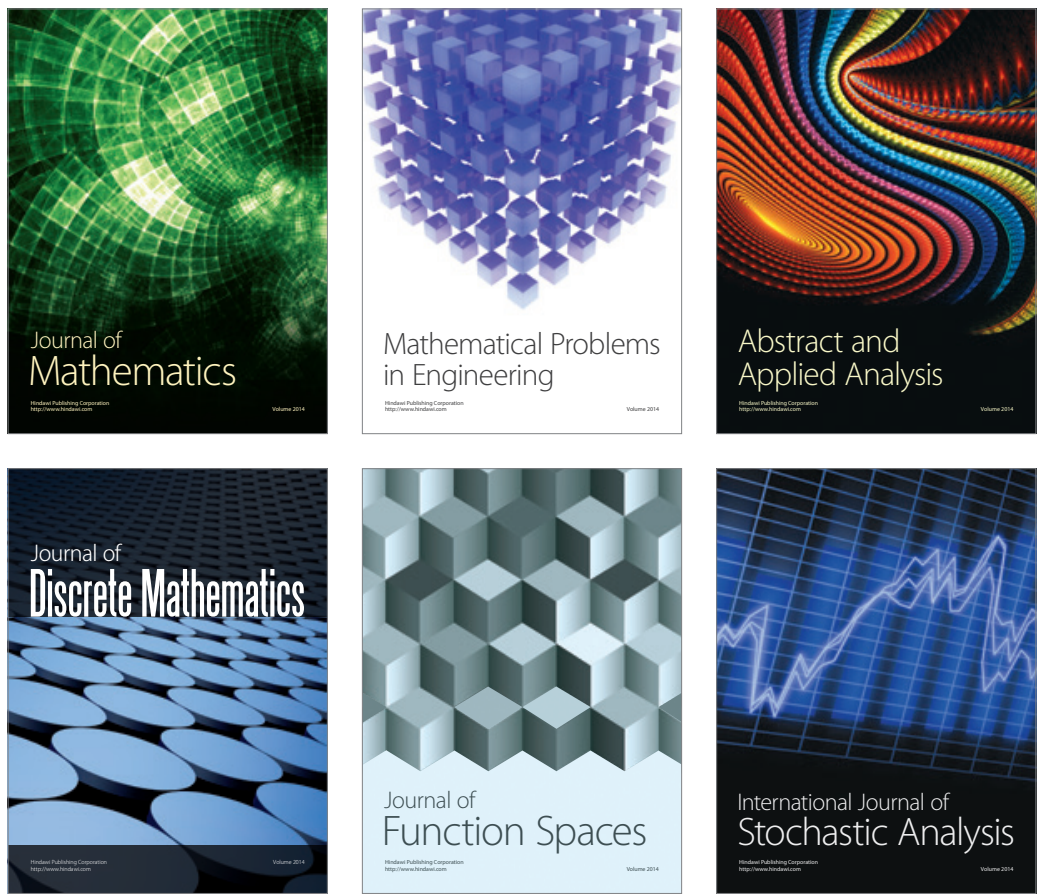

Journal of

Function Spaces

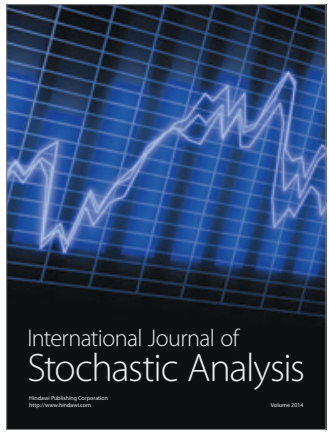

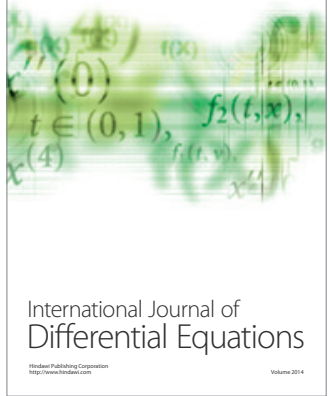
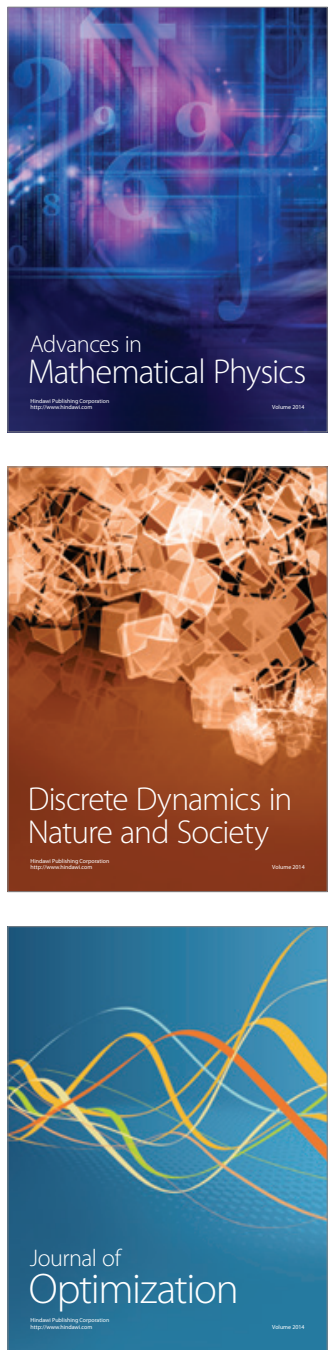